Article

\title{
Antioxidant Status, Blood Constituents and Immune Response of Broiler Chickens Fed Two Types of Diets with or without Different Concentrations of Active Yeast
}

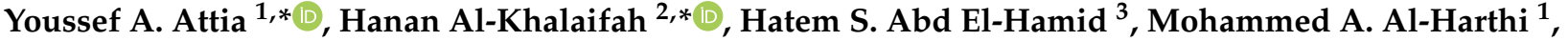 \\ Salem R. Alyileili ${ }^{4}$ and Ali A. El-Shafey ${ }^{5}$
}

Citation: Attia, Y.A.; Al-Khalaifah, H.; Abd El-Hamid, H.S.; Al-Harthi, M.A.; Alyileili, S.R.; El-Shafey, A.A.

Antioxidant Status, Blood

Constituents and Immune Response of Broiler Chickens Fed Two Types of Diets with or without Different Concentrations of Active Yeast.

Animals 2022, 12, 453.

https://doi.org/10.3390/

ani12040453

Academic Editor: Rosalia Crupi

Received: 9 November 2021

Accepted: 9 February 2022

Published: 12 February 2022

Publisher's Note: MDPI stays neutral with regard to jurisdictional claims in published maps and institutional affiliations.

Copyright: (C) 2022 by the authors. Licensee MDPI, Basel, Switzerland. This article is an open access article distributed under the terms and conditions of the Creative Commons Attribution (CC BY) license (https:// creativecommons.org/licenses/by/ $4.0 /)$.
1 Agriculture Department, Faculty of Environmental Science, King Abdulaziz University, P.O. Box 80208, Jeddah 21589, Saudi Arabia; malharthi@kau.edu.sa

2 Environment and Life Sciences Research Center, Kuwait Institute for Scientific Research (KISR), P.O. Box 24885, Safat-Kuwait 13109, Kuwait

3 Poultry and Fish Diseases Department, Faculty of Veterinary Medicine, Damanhour University, Damanhour 22511, Egypt; drhatem_deltavet@yahoo.co.uk

4 Department of Integrative Agriculture, College of Food and Agriculture, United Arab Emirates University, Al-Ain 15551, United Arab Emirates; salem7178767@gmail.com

5 Animal and Poultry Production Department, Faculty of Agriculture, Damanhour University, Damanhour 22511, Egypt; alielshafey2015@gmail.com

* Correspondence: yaattia@kau.edu.sa (Y.A.A.); hkhalifa@kisr.edu.kw (H.A.-K.); Tel.:+966-568575961 (Y.A.A.); +965-94949325 (H.A.-K.)

Simple Summary: Rations for broilers can be safely supplemented with probiotics such as active Saccharomyces cerevisiae (SC) yeast to stimulate oxidative reactions and immune response against stress and infectious agents. The current study suggested that SC yeast enhanced antioxidant capacity, growth rate, immune organ weights, immune response and the survival rate of broilers after Avian Influenza virus challenge at 38 days of age.

Abstract: Probiotics, such as active yeasts, are widely used to enhance poultry production and reduce feeding costs. This study aimed to investigate the antioxidant and immune responses of broilers to different concentrations of active Saccharomyces cerevisiae (SC) when supplemented to two types of diets. A total of 216 1-day-old Arbor Acres unsexed chicks were used in a factorial design, involving two feeds (regular- versus low-density diet) and three concentrations of SC ( $\% \%, 0.02 \%$ and $0.04 \%)$. The results revealed that the low-density diet reduced the body weight and production index of broilers. The addition of SC improved the production index more than the control diet. Total antioxidant capacity (TAC), alanine aminotransferase (ALT), aspartate aminotransferase (AST) and eosinophils were significantly higher in response to the regular-density diet than the low-density diet; however, phagocytic activity (PA), lymphocyte and lysozyme activity (LYS) were lower. Saccharomyces cerevisiae reduced ALT, AST, malondialdehyde (MAD) and TAC more than the standard set, but improved packed cell volume (PCV), hemoglobin $(\mathrm{Hgb})$, red blood cells (RBCs), lymphocytes, monocytes, heterophils, phagocytic index (PI) and the immune response to Newcastle disease virus (NDV) and avian influenza (AI). In conclusion, supplementation of a regular- or low-density diet with SC at a concentration of $0.02 \%$ or $0.04 \%$ improved the antioxidant parameters, immune status and production index of broilers against stress and infectious agents.

Keywords: active yeast; antioxidant status; broilers; nutrient density; immune response

\section{Introduction}

There is a growing interest in the innovative biofortification of poultry feed rations through the use of functional ingredients to improve feed utilization and enhance production performance and the immune status of the flocks [1-8]. 
Saccharomyces cerevisiae (SC) has been used in poultry feed rations to enhance resistance to aflatoxicosis in poultry [9]. The antioxidant status and capacity of poultry have been shown to be significantly enhanced by supplementing poultry feed rations with SC, either alone or in combination with probiotics $[10,11]$. The weights of the primary and secondary immune organs in broilers have been shown to be increased after dietary supplementation with active yeast, potentially indicating immunocompetence in broilers [12]. In addition, Kiarie et al. [13] revealed that when added with feed enzymes, yeast derivatives can modulate cellular- and humoral-mediated immunity in broilers against intestinal coccidial infections. Zhou et al. [14] investigated the ability of yeast fractions to prevent pullorum disease and fowl typhoid in breeders. The results of the study revealed that dietary fortification of yeast fractions significantly reduced disease infection in the challenged breeders. In the same study, the positive culling rate of the pullets and their body weight were significantly reduced because of the addition of yeast fractions to the feed of birds challenged with Salmonella infection [14]. The intestinal microflora balance has also been shown to be improved in birds with dietary supplementation of yeast, due to the presence of mannan-oligosaccharides and fructo-oligosaccharides in the cell wall of yeast [15-19]. However, debate remains regarding the effects of active yeast on the production performance and immune status of chickens, particularly when they are fed diets containing different nutrient profiles or are placed under environmental stress [20-23]. Hayat et al. [24] suggested that this could be due to genetic differences, or differences in species, age, or environmental conditions. Thus, this study was carried out to fill this knowledge gap.

The current study aimed to fill the gap in the literature about the response of broilers fed two types of diets, with or without different concentrations of SC (active yeast) with respect to antioxidant status, blood constituents and immune status. Although previous studies investigated the effect of SC on the productive performance parameters in broiler chickens, there are relatively limited data in the literature on the direct effect of SC probiotic on the antioxidative status, blood constituents and immune status in broiler chickens fed low-density diet. The current literature does not yet adequately address the approaches of nutrient manipulation in broiler feed rations to shed light on the relationship between the effect of SC in low-density diets and the immune response of broiler chickens. Accordingly, this study was executed to elaborate on this vital relationship.

\section{Materials and Methods}

\subsection{Birds, Dietary Treatments, Experimental Plan and Housing}

This research work was approved by the Deanship of Scientific Research, King Abdulaziz University, Saudi Arabia, under protocol no: (FP-217-42 H). The protocol recommends general humane treatment of animals that did not cause animal (s) pain, suffering, distress, or lasting harm, according to the Royal Decree number M59 in 14/9/1431H.

A total of 216, 1-day-old Arbor Acres broilers (mixed sexes) were acquired. The chicks were marked randomly by way of wing-banding and were housed in 36 pens with 6 birds per pen (replicate). Each treatment involved 6 replicates. The body weight (BW) of all chicks was similar at the start of the experiment.

The chickens were provided mash feeds ad libitum, along with freely accessed waterers. During the first 7 days, $23 \mathrm{~h}$ of light were used, followed by $20 \mathrm{~h}$ of light until the end of the experiment.

A factorial design $(2 \times 3)$ was applied to the experiment using two diets (a regular versus a low-density diet containing 10\% fewer nutrients than the regular diet) and three levels of SC (an unfortified standard, $0.02 \%$, or $0.04 \%$ SC). The broiler feed rations were formulated based on the Arbor Acres broilers guide [25]. There were six replications in each treatment and each replicate involved six birds. The SC yeast was purchased from China Way Co-operation, Taiwan, and had 12,000,000,000 active yeast per gram. The optimal dosage of SC was 200 to $400 \mathrm{~g}$ per ton of feed. Table 1 shows the composition of the dietary treatments fed to the chickens. 
Table 1. Basal chemical composition of the experimental diets.

\begin{tabular}{|c|c|c|c|c|c|c|}
\hline \multirow{2}{*}{ Ingredients (\%) } & \multicolumn{3}{|c|}{ Regular-Density Diet } & \multicolumn{3}{|c|}{ Low-Density Diet } \\
\hline & Starter & Grower & Finisher & Starter & Grower & Finisher \\
\hline Maize & 51.27 & 52.02 & 55.85 & 46.01 & 46.61 & 50.40 \\
\hline Rye & 0 & 5 & 7 & 0 & 4.48 & 6.2 \\
\hline Wheat bran & 0 & 0 & 0 & 10 & 10 & 10 \\
\hline Soybean meal (44\% CP) & 32.8 & 24.4 & 28.0 & 29.3 & 21.9 & 25.0 \\
\hline Vegetable oil & 2.25 & 2.0 & 5.3 & 2.01 & 1.79 & 4.7 \\
\hline Full-fat soybean meal & 10 & 13 & 1.6 & 9 & 11.64 & 1.42 \\
\hline Dicalcium phosphate & 1.8 & 1.6 & 1.0 & 1.8 & 1.6 & 1.0 \\
\hline Limestone & 1.0 & 1.0 & 0.15 & 1.0 & 1.0 & 0.15 \\
\hline L-Lysine & 0.10 & 0.15 & 0.2 & 0.10 & 0.15 & 0.2 \\
\hline DL-Methionine & 0.15 & 0.20 & 0.30 & 0.15 & 0.20 & 0.30 \\
\hline Vit $+\min$ premix $^{1}$ & 0.30 & 0.30 & 0.30 & 0.30 & 0.30 & 0.30 \\
\hline $\mathrm{NaCl}$ & 0.30 & 0.30 & 0.30 & 0.30 & 0.30 & 0.30 \\
\hline Washed building sand & 0.03 & 0.03 & 0.00 & 0.03 & 0.03 & 0.03 \\
\hline Total & 100 & 100 & 100 & 100 & 100 & 100 \\
\hline \multicolumn{7}{|c|}{ Calculated and deterimed analyses } \\
\hline $\begin{array}{l}\text { Metabolizable Energy } \\
(\mathrm{kcal} / \mathrm{kg})^{2}\end{array}$ & 3038 & 3100 & 3196 & 2735 & 2790 & 2876 \\
\hline Crude Protein $(\%)^{3}$ & 22.8 & 21.2 & 18.5 & 20.5 & 19.1 & 16.7 \\
\hline Lysine $(\%)^{2}$ & 1.33 & 1.23 & 1.04 & 1.20 & 1.11 & 0.94 \\
\hline Methionine $(\%)^{2}$ & 0.50 & 0.52 & 0.48 & 0.45 & 0.47 & 0.43 \\
\hline Meth + cysteine $(\%)^{2}$ & 0.87 & 0.87 & 0.78 & 0.78 & 0.78 & 0.70 \\
\hline Calcium $(\%)^{2}$ & 0.91 & 0.85 & 0.83 & 0.82 & 0.77 & 0.75 \\
\hline Av. P $(\%)^{2}$ & 0.46 & 0.41 & 0.41 & 0.414 & 0.369 & 0.369 \\
\hline Crude fat $(\%)^{3}$ & 6.09 & 6.45 & 6.8 & 5.48 & 5.81 & 6.12 \\
\hline Crude fibre $(\%)^{3}$ & 3.55 & 4.48 & 4.75 & 3.20 & 4.03 & 4.28 \\
\hline $\operatorname{Ash}(\%)^{3}$ & 5.22 & 5.48 & 5.71 & 4.70 & 4.93 & 5.14 \\
\hline
\end{tabular}

${ }^{1}$ Vit + Min mix. contains the following nutrients. Values are per kilogram of the diet: Vit. A, 12,000 IU; Vit. E (dl- $\alpha$-tocopheryl acetate), $20 \mathrm{mg}$; menadione, $2.3 \mathrm{mg}$; Vit. $\mathrm{D}_{3}$, $2200 \mathrm{ICU}$; riboflavin, $5.5 \mathrm{mg}$; calcium pantothenate, $12 \mathrm{mg}$; nicotinic acid, $50 \mathrm{mg}$; Choline, $250 \mathrm{mg}$; Vit. $\mathrm{B}_{12}, 10 \mu \mathrm{g}$; Vit. $\mathrm{B}_{6}, 3 \mathrm{mg}$; thiamine $3 \mathrm{mg}$; folic acid, $1 \mathrm{mg}$ d-biotin, $0.05 \mathrm{mg}$; Trace minerals (mg/kg of diet): $\mathrm{Mn}, 80 ; \mathrm{Zn}, 60 ; \mathrm{Fe}, 35 ; \mathrm{Cu}$, 8; Selenium, $0.1 \mathrm{mg},{ }^{2}$ Calculated analyses, ${ }^{3}$ Determined analyses.

\subsection{Data Gathering}

Average pen body weight $(\mathrm{g})$ was recorded at 1,21 and 38 days of age and used to calculate the body weight gain (BWG, g/bird). The average pen feed intake (g/bird) was recorded and used to calculate the feed conversion rate (FCR, $\mathrm{g}$ feed/g gain) and survival rate (100-mortality rate) during the following periods: $1-21$ days, $22-38$ days and 1-38 days of age. The production index was calculated as follows: $\mathrm{BW}(\mathrm{kg}) \times$ survival rate Production index $=\times 100$ production period in days $\times$ FCR.

\subsection{Blood Sampling}

Blood was collected from each treatment group $(n=6)$ before vaccination and again at 8 days post-vaccination. The serum was harvested by centrifuging the blood at $1500 \times g$ for $15 \mathrm{~min}$.

\subsection{Antioxidant Status and Biochemical Traits}

Serum total antioxidant capacity (TAC) and malondialdehyde (MAD) were assayed as described in Erel [26] and Wyatt et al. [27], respectively. They were determined using commercial kits produced by Diamond Diagnostics (23 EL-Montazah St. Heliopolis, Cairo, Egypt, http:/ / www.diamonddiagnostics.com (accessed on 1 February 2022). Total plasma protein and albumin concentrations were measured using the methods outlined in Armstrong and Carr [28] and Doumas and Peters [29], respectively. Subtracting albumin concentration from serum total protein gives an estimate of the globulin concentration, as described in Giangiacomo et al. [30]. Various kinds of globulin ( $\alpha-, \beta$ - and $\gamma$-globulin) 
were determined based on methods described in Elias [31]. The activities of alanine aminotransferase (ALT, U/L) and aspartate aminotransferase (AST, U/L) were determined using techniques described in Reitman and Frankel [32]. Alkaline phosphatase (ALKP) enzyme action was measured in plasma, as described by Kim and Wyckoff [33].

\subsection{Hematological Parameters}

Wintrobe hematocrit tubes were used to measure the packed cell volume (PCV, \%) by centrifugation for $20 \mathrm{~min}$. at $2000 \times \mathrm{g}$. Hemoglobin $(\mathrm{Hgb})$ level was estimated using the technique described in Eilers [34]. The mean corpuscular volume (MCV, $\mu \mathrm{m} 3)$, mean corpuscular hemoglobin $(\mathrm{MCH}, \mathrm{Pg})$ and mean corpuscular hemoglobin concentration (MCHC, g/dL) were measured using the equations described in Jain [35].

\subsection{Immune Indices}

The phagocytic activity (PA, \% of phagocytic cells engulfing yeast cells) and phagocytic index (PI, number of yeast cell phagocytized/number of phagocytic cells) were determined as described in Kawahara et al. [36].

Broiler chickens were vaccinated according to the following schedule: inactivated avian influenza (AI) H5N2 at 10 days of age. Chickens were vaccinated with clone 30 eye drop on day 8 for Newcastle disease (NDV) and bivalent NDV vaccine was administered underneath the neck membrane, simultaneously with clone 30 , at 8 days of age. The Gumboro intermediate vaccine and clone 30 were administered at 12 and 21 days of age, respectively (Nobilis, Intervet, Boxmeer, The Netherlands).

Blood samples ( $n=6$ per group) were taken just before vaccination ( 0 days postvaccination) and again on 8th day post-vaccination. The samples were centrifuged at $1500 \times g$ for 15 min for serum separation, to determine antibody titers against NDV via the hemagglutination inhibition test (HI) test. This test was done using hemagglutination inhibition (HI) test according to OIE [37]. The geometric mean titer was calculated as recommended by the World Organization for Animal Health (OIE) [38].

Antibody responses were determined by the HI test, according to Seal et al. [39]. The assay measures antibodies attached to influenza antigen-coated plates [40]. Hemagglutination inhibition for NDV and AI were measured as described in Takatsy and Hamar [41].

A lymphocyte transformation test was performed, as described in Balhaa et al. [42]. Lympholyte-H (Cedarlane Laboratories Ltd., Burlington, ON, Canada) was used to layer the collected heparinized blood. After centrifugation, the lymphocytes in the interface layer were collected, washed and suspended in culture medium.

Serum bactericidal activity to the Aeromonas hydrophila strain was conducted following the protocols described in Rainger and Rowley [43]. The turbidimetric method was used to measure serum lysozyme activity [44]. The results were reflected as one unit of lysozyme activity as a reduction in absorbency of $0.001 / \mathrm{min}$ Lysozyme activity $=(\mathrm{A} 0-\mathrm{A}) / \mathrm{A}$.

\subsection{Challenge Test}

The challenge test was conducted to study the impact of the diet on the survival rate of chicks $[45,46]$ between 38 and 48 days of age. Six broiler chickens per treatment were randomly selected at 38 days of to represent all treatment replications. The chickens were vaccinated with inactivated avian influenza (AI) H5N2 at 10 days of age and then challenged with $\mathrm{H} 5 \mathrm{~N} 1$ at 38 days via the oculo-nasal route with $0.2 \mathrm{~mL} /$ bird $\left(10^{6} /\right.$ dose). The H5N1 was from research laboratory of Poultry Disease, Fac. Vet. Med., Damanhour University, where the challenge test was carried out following the regulations for animal welfare approved by the authorized ethics committee of the Egyptian Ministry of Agriculture according to Decree No. 27, 1967. The mortality was recorded daily during 38-48 days of age.

\subsection{Histopathological Study}

On day 38, 6 chickens from each treatment replicate were randomly selected and euthanized under anesthesia via an intravenous injection of sodium pentobarbital $(50 \mathrm{mg} / \mathrm{kg}$; 
CAMEO chemicals, Tampa, FL, USA). Necropsies were performed for sample collection. Lymphoid organs (bursa of Fabricius, thymus and spleen) were weighed, and the body weight ratios of organs were calculated.

In addition, intestine, bursa of Fabricious, thymus and spleen specimens were collected from randomly collected broilers ( $n=6$ per treatment) at 38 days of age. Tissue specimens were prepared as previously described by Culling [47].

\subsection{Morphometrical Study}

An Optika binocular microscope, with an Optika imaging analyzer, was used to examine the morphological appearance of intestinal villi, determine the absorption surface and measure the longitudinal axis of the large follicle of the bursa. Five segments from each bird were used for this examination. In addition, quantitative measurements of the thymus cortical: medullary ratio were performed and the hyperplasia of the lymphoblastic cells was assayed by examining the spleen. The scale used was as follows: $(-)$ for weak hyperplasia; $(+)$ for moderate hyperplasia; (++) for severe hyperplasia.

\subsection{Statistical Analyses}

The data were analyzed using general linear models in SAS (SAS Institute, Cary, NC, USA [48]). A two-way factorial design (two kinds of diets $\times$ three concentrations of SC) was used to analyze the effects of the treatments on most of the parameters. An exception was survival rate in the challenge study, where age was included as a main effect only. The replicate was the experimental unit. Data were arcsine transformed prior to analysis to improve normality. Student-Newman-Keuls (SNK) post hoc tests were applied to evaluate differences between factor levels in the model. Differences were considered significant if $p \leq 0.05$.

\section{Results}

\subsection{Growth Performance}

Table 2 shows the impact of yeast concentrations on body weight and the European Production Efficiency Index (EPEI) of broilers fed regular- and low-density diets. During the experiment, the low-density diet was found to decrease the final body weight and EPEI, reaching $5 \%$ over the duration of the study. Diets supplemented with $0.02 \%$ and $0.04 \%$ SC resulted in a production index that was significantly enhanced relative to the control diet. However, the addition of SC in the diet at a concentration of $0.04 \%$ had a more substantial effect on the growth of 38-day-old chickens than the $0.02 \%$ level (Table 2). There was no significant relationship between the amount of SC and diet on the growth of broilers or EPEI between 1 and 38 days of age.

Table 2. Impact of different concentrations of Saccharomyces cerevisiae on body weight, survival rate, European Production Efficiency Index and blood hematological parameters of broilers fed regular- or low-density diets from days 1 to 38 of age.

\begin{tabular}{|c|c|c|c|c|c|c|c|c|c|}
\hline Treatment & $\begin{array}{c}\text { Body Weight } \\
\text { at } 38 \text { Days of } \\
\text { Age, } g\end{array}$ & $\begin{array}{c}\text { Survival } \\
\text { Rate, } \%\end{array}$ & $\begin{array}{l}\text { European } \\
\text { Production } \\
\text { Efficiency } \\
\text { Index }\end{array}$ & PCV, \% & $\begin{array}{l}\text { Hemoglobin, } \\
\text { g/dL }\end{array}$ & $\begin{array}{r}\text { RBC, } \\
10^{6} / \mathrm{uL}\end{array}$ & $\mathrm{MCV}, \mathrm{fL}$ & $\mathrm{MCH}, \mathrm{pg}$ & $\begin{array}{l}\mathrm{MCHC}, \\
\mathrm{g} / \mathrm{dL}\end{array}$ \\
\hline \multicolumn{10}{|c|}{ Effect of diet } \\
\hline $\begin{array}{l}\text { Regular } \\
\text { density }\end{array}$ & $2099^{a}$ & 100 & $314^{\mathrm{a}}$ & 30.4 & 9.91 & 1.65 & 186 & 60.6 & 32.7 \\
\hline Low density & $2004^{b}$ & 100 & $297^{b}$ & 30.0 & 10.0 & 1.72 & 177 & 58.7 & 33.3 \\
\hline \multicolumn{10}{|c|}{ Effect of yeast concentration } \\
\hline Control & $1865^{c}$ & 100 & $281^{\mathrm{b}}$ & $28.3^{b}$ & $9.43^{b}$ & $1.60^{\mathrm{b}}$ & 180 & 59.7 & 33.2 \\
\hline $0.02 \%$ & $2106^{b}$ & 100 & $318^{a}$ & $31.6^{\mathrm{a}}$ & $10.5^{\mathrm{a}}$ & $1.76^{\mathrm{a}}$ & 182 & 60.8 & 33.5 \\
\hline $0.04 \%$ & $2183^{a}$ & 100 & $318^{a}$ & $30.7^{\mathrm{a}}$ & $9.87^{b}$ & $1.70^{a b}$ & 182 & 58.5 & 32.2 \\
\hline
\end{tabular}


Table 2. Cont.

\begin{tabular}{|c|c|c|c|c|c|c|c|c|c|c|}
\hline \multicolumn{2}{|c|}{ Treatment } & $\begin{array}{c}\text { Body Weight } \\
\text { at } 38 \text { Days of } \\
\text { Age, g }\end{array}$ & $\begin{array}{c}\text { Survival } \\
\text { Rate, \% }\end{array}$ & $\begin{array}{l}\text { European } \\
\text { Production } \\
\text { Efficiency } \\
\text { Index }\end{array}$ & PCV, \% & $\begin{array}{l}\text { Hemoglobin, } \\
\text { g/dL }\end{array}$ & $\begin{array}{r}\text { RBC, } \\
10^{6} / \mathrm{uL}\end{array}$ & MCV, fL & $\mathrm{MCH}, \mathrm{pg}$ & $\begin{array}{c}\text { MCHC, } \\
\text { g/dL }\end{array}$ \\
\hline \multicolumn{11}{|c|}{ Interaction between diet type and yeast concentration } \\
\hline Regular & Control & 1946 & 100 & 288 & $18.7^{\mathrm{b}}$ & $8.75^{\mathrm{b}}$ & 1.58 & $172^{b, c}$ & $55.7^{\mathrm{b}, \mathrm{c}}$ & 32.4 \\
\hline den- & $0.02 \%$ & 2171 & 100 & 321 & $32.0^{\mathrm{a}}$ & $10.6^{\mathrm{a}}$ & 1.75 & $185^{\mathrm{a}, \mathrm{b}, \mathrm{c}}$ & $61.6^{\mathrm{a}, \mathrm{b}}$ & 33.4 \\
\hline sity & $0.04 \%$ & 2179 & 100 & 334 & $32.3^{\mathrm{a}}$ & $10.3^{\mathrm{a}}$ & 1.62 & $202^{\mathrm{a}}$ & $64.5^{\mathrm{a}}$ & 32.2 \\
\hline Low & Control & 1784 & 100 & 274 & $29.7^{a}$ & $10.1^{\mathrm{a}}$ & 1.61 & $188^{a, b}$ & $63.8^{a, b}$ & 34.0 \\
\hline den- & $0.02 \%$ & 2041 & 100 & 315 & $31.3^{a}$ & $10.5^{a}$ & 1.77 & $179^{a, b, c}$ & $60.0^{a, b}$ & 33.5 \\
\hline sity & $0.04 \%$ & 2187 & 100 & 303 & $29.1^{a}$ & $9.37^{b}$ & 1.78 & $163^{c}$ & $52.4^{c}$ & 32.3 \\
\hline \multicolumn{2}{|c|}{ SEM } & 41.30 & ND & 9.67 & 0.955 & 0.316 & 0.058 & 9.99 & 3.09 & 1.02 \\
\hline \multirow{3}{*}{\multicolumn{2}{|c|}{$\begin{array}{c}\text { Diet type } \\
\text { Yeast } \\
\text { Interaction }\end{array}$}} & 0.0008 & ND & 0.0408 & $\begin{array}{c}p \text { value } \\
0.6336\end{array}$ & 0.7485 & 0.1473 & 0.256 & 0.466 & 0.458 \\
\hline & & 0.0001 & ND & 0.0006 & 0.0040 & 0.0038 & 0.0273 & 0.966 & 0.753 & 0.442 \\
\hline & & 0.1182 & ND & 0.4298 & 0.0121 & 0.0021 & 0.4088 & 0.029 & 0.009 & 0.715 \\
\hline
\end{tabular}

a,b,c Means within a column with different superscripts are significantly different based on Student-NewmanKeuls (SNK) post hoc tests. MCV = Mean corpuscular volume; $\mathrm{MCH}=$ Mean corpuscular hemoglobin $\mathrm{MCHC}=$ Mean corpuscular hemoglobin concentration; Number of observations was 6 replicates per interaction cell. ND $=$ Not done.

\subsection{Antioxidant Status and Biochemical Traits}

Tables 3 and 4 show the impact of different yeast concentrations on the liver enzyme index, peroxidation index and blood serum biochemical constituents of broilers fed regular and low-density diets from 1 to 38 of age.

Table 3. Impact of different concentrations of Saccharomyces cerevisiae on liver enzymes and malondialdehyde (MDA) of broilers fed regular- and low-density diets from days 1 to 38 of age.

\begin{tabular}{|c|c|c|c|c|c|c|}
\hline \multicolumn{2}{|c|}{ Treatment } & AST (U/L) & ALT (U/L) & $\begin{array}{c}\text { AST/ALT } \\
\text { Ratio }\end{array}$ & $\begin{array}{l}\text { Alkaline } \\
\text { Phos- } \\
\text { phatase } \\
\text { (U/L) }\end{array}$ & $\begin{array}{l}\text { MDA.m } \\
\text { Mol/dL }\end{array}$ \\
\hline \multirow{3}{*}{\multicolumn{2}{|c|}{$\begin{array}{l}\text { Regular density } \\
\text { Low density }\end{array}$}} & \multicolumn{3}{|c|}{ Effect of diet } & \multirow[b]{2}{*}{11.4} & \multirow[b]{2}{*}{1.44} \\
\hline & & $64.7^{\mathrm{a}}$ & $56.4^{\mathrm{a}}$ & 1.14 & & \\
\hline & & $63.7^{\mathrm{b}}$ & $55.1^{\mathrm{b}}$ & 1.15 & 11.6 & 1.45 \\
\hline \multicolumn{7}{|c|}{ Effect of yeast concentration } \\
\hline \multicolumn{2}{|c|}{ Control } & $66.0^{\mathrm{a}}$ & $57.8^{\mathrm{a}}$ & 1.14 & 11.5 & $1.57^{\mathrm{a}}$ \\
\hline \multicolumn{2}{|c|}{$0.02 \%$} & $63.43^{b}$ & $54.9^{b}$ & 1.15 & 11.2 & $1.32^{\mathrm{c}}$ \\
\hline \multicolumn{2}{|c|}{$0.04 \%$} & $63.2^{\mathrm{b}}$ & $54.6^{\mathrm{b}}$ & 1.15 & 11.8 & $1.45^{\mathrm{b}}$ \\
\hline \multicolumn{7}{|c|}{ Interaction between diet and yeast concentration } \\
\hline \multirow{3}{*}{$\begin{array}{l}\text { Regular } \\
\text { density }\end{array}$} & Control & $65.5^{\mathrm{a}, \mathrm{b}}$ & $57.1^{\mathrm{a}, \mathrm{b}}$ & 1.14 & 11.6 & $1.41^{\mathrm{b}}$ \\
\hline & $0.02 \%$ & $64.2^{\mathrm{b}}$ & $56.1^{\mathrm{b}}$ & 1.14 & 11.2 & $1.43^{b}$ \\
\hline & $0.04 \%$ & $64.5^{b}$ & $56.1^{b}$ & 1.14 & 11.3 & $1.47^{\mathrm{b}}$ \\
\hline \multirow{3}{*}{$\begin{array}{c}\text { Low } \\
\text { density }\end{array}$} & Control & $66.6^{\mathrm{a}}$ & $58.6^{\mathrm{a}}$ & 1.13 & 11.3 & $1.73^{\mathrm{a}}$ \\
\hline & $0.02 \%$ & $62.6^{\mathrm{c}}$ & $53.7^{c}$ & 1.16 & 11.2 & $1.21^{\mathrm{c}}$ \\
\hline & $0.04 \%$ & $62.0^{\mathrm{c}}$ & $53.1^{\mathrm{c}}$ & 1.16 & 12.2 & $1.42^{b}$ \\
\hline \multicolumn{2}{|c|}{ SEM } & 0.537 & 0.701 & 0.015 & 0.587 & 0.058 \\
\hline & & $p$ value & & & \\
\hline & & 0.0283 & 0.0299 & 0.4385 & 0.6668 & 0.7284 \\
\hline \multicolumn{2}{|c|}{ Yeast concentration } & 0.0001 & 0.0001 & 0.5854 & 0.6351 & 0.0005 \\
\hline \multicolumn{2}{|c|}{ Interaction } & 0.0046 & 0.0052 & 0.5946 & 0.6077 & 0.0001 \\
\hline
\end{tabular}

a,b,c Means within a column with different superscripts are significantly different based on StudentNewman-Keuls (SNK) post hoc test. ALT = Alanine aminotransferase; AST = Aspartate aminotransferase AST $/$ ALT $=$ Aspartate aminotransferase to alanine aminotransferase ratio; MAD = Malondialdehyde. Number of observations was 6 per interaction cell. 
Table 4. Impact of different concentrations of Saccharomyces cerevisiae on the biochemical constituents of blood serum of broilers fed regular- and low-density diets from days 1 to 38 of age.

\begin{tabular}{|c|c|c|c|c|c|c|c|c|}
\hline \multirow{2}{*}{\multicolumn{2}{|c|}{ Treatment }} & \multicolumn{7}{|c|}{ Serum Biochemical Constituents (mg/dL) } \\
\hline & & $\begin{array}{c}\text { Total } \\
\text { Protein, g/dL }\end{array}$ & $\begin{array}{l}\text { Albumin, } \\
\text { g/dL }\end{array}$ & \multirow{2}{*}{$\begin{array}{l}\begin{array}{c}\text { Globulin, } \\
\text { g/dL }\end{array} \\
\text { Effect of die }\end{array}$} & \multirow[t]{2}{*}{$\begin{array}{c}\alpha-\text {-Gloulin, } \\
\text { g/dL }\end{array}$} & \multirow[t]{2}{*}{$\begin{array}{c}\beta \text {-Globulin, } \\
\text { g/dL }\end{array}$} & \multirow[t]{2}{*}{$\begin{array}{c}\gamma \text {-Globulin, } \\
\text { g/dL }\end{array}$} & GAR \\
\hline \multirow{3}{*}{\multicolumn{2}{|c|}{$\begin{array}{l}\text { Regular density } \\
\text { Low density }\end{array}$}} & & & & & & & \\
\hline & & 5.00 & 2.70 & 2.24 & 0.912 & 0.730 & $0.595^{\mathrm{a}}$ & 0.846 \\
\hline & & 4.80 & 2.73 & 2.11 & 0.958 & 0.720 & $0.437^{\mathrm{b}}$ & 0.784 \\
\hline \multicolumn{9}{|c|}{ Effect of yeast concentration } \\
\hline \multirow{3}{*}{\multicolumn{2}{|c|}{$\begin{array}{c}\text { Control } \\
0.02 \% \\
0.04 \%\end{array}$}} & $4.73^{\mathrm{b}}$ & 2.64 & 2.09 & 0.837 & $0.656^{\mathrm{b}}$ & 0.600 & 0.828 \\
\hline & & $5.01^{\mathrm{a}}$ & 2.70 & 2.17 & 0.956 & $0.768^{\mathrm{a}}$ & 0.450 & 0.792 \\
\hline & & $5.00^{\mathrm{a}}$ & 2.81 & 2.26 & 1.01 & $0.756^{\mathrm{a}}$ & 0.500 & 0.824 \\
\hline \multicolumn{9}{|c|}{ Interaction between diet and yeast concentration } \\
\hline \multirow{3}{*}{$\begin{array}{l}\text { Regular } \\
\text { density }\end{array}$} & Control & 4.78 & 2.63 & 2.15 & 0.837 & 0.637 & 0.675 & 0.870 \\
\hline & $0.02 \%$ & 4.86 & 2.75 & 2.26 & 0.962 & 0.800 & 0.500 & 0.820 \\
\hline & $0.04 \%$ & 5.10 & 2.72 & 2.31 & 0.837 & 0.762 & 0.612 & 0.846 \\
\hline \multirow{3}{*}{$\begin{array}{c}\text { Low } \\
\text { density }\end{array}$} & Control & 4.68 & 2.65 & 2.03 & 0.837 & 0.675 & 0.525 & 0.786 \\
\hline & $0.02 \%$ & 4.86 & 2.65 & 2.08 & 0.950 & 0.737 & 0.400 & 0.763 \\
\hline & $0.04 \%$ & 5.02 & 2.91 & 2.22 & 1.087 & 0.750 & 0.387 & 0.802 \\
\hline \multicolumn{2}{|c|}{ SEM } & 0.137 & 0.153 & 0.113 & 0.072 & 0.044 & 0.067 & 0.075 \\
\hline & & & $p$ value & & & & \\
\hline & Diet & 0.191 & 0.792 & 0.1845 & 0.4459 & 0.7356 & 0.0066 & 0.3243 \\
\hline \multicolumn{2}{|c|}{ Yeast } & 0.0312 & 0.5149 & 0.3136 & 0.0612 & 0.0323 & 0.0908 & 0.8728 \\
\hline \multicolumn{2}{|c|}{ Interaction } & 0.8182 & 0.6448 & 0.9241 & 0.4702 & 0.5448 & 0.6516 & 0.9641 \\
\hline
\end{tabular}

a,b Means within a column with different superscripts are significantly different based on Student-Newman-Keuls (SNK) post hoc tests. GAR = Globulin to albumin ratio. Number of observations was 6 chicks per interaction cell.

Data for the biochemical components of blood serum show no significant impact of diet type on serum biochemical constituents (total protein, albumin, $\alpha-, \beta$-globulin, globulin and globulin/albumin ratio). However, $\gamma$-globulin, AST and ALT were significantly lower in birds fed a low-density diet than a regular-density diet (Tables 3 and 4). Supplementation of the diet with SC at $0.02 \mathrm{~g} / \mathrm{kg}$ and $0.04 \%$ significantly lowered serum AST, ALT and MAD relative to the standard. Additionally, total serum protein and $\beta$ - globulin were significantly greater in the groups that received SC supplementation compared with control groups without SC. There was no interaction effect between SC level and dietary treatment on blood biochemical constituents (ALT/AST and alkaline phosphatase, Table 3; and total protein, albumin, $\alpha, \beta$ and $\delta$-globulin, G/A ratio, Table 4 ). However, there was a significant impact of the interaction on serum AST, ALT and MAD. Supplementation with SC at both concentrations significantly decreased serum AST and ALT of broilers on the low-density diet compared to the regular-density diet. Additionally, $0.02 \%$ of SC decreased MAD in broilers fed a low-density diet compared to a regular density diet.

\subsection{Hematology of Blood}

Table 5 shows the effects of different concentrations of yeast on white blood cells and its subpopulations of broilers fed regular- and low-density diets from 1 to 38 days of age. The results showed that lymphocytes of broilers fed a low-density diet were significantly higher than that of broilers fed a regular-density diet, but eosinophils were lower. The addition of SC to the diet greatly enhanced PCV, lymphocytes and monocytes; and $0.02 \%$ SC significantly increased $\mathrm{Hgb}$ and $\mathrm{RBCs}$, but decreased heterophils and the $\mathrm{H} / \mathrm{L}$ ratio, relative to control diet (Table 5). However, the addition of SC to the diet at $0.04 \%$ significantly increased heterophils. 
Table 5. Impact of different concentrations of Saccharomyces cerevisiae on white blood cells (WBC) and its subpopulations in broilers fed regular- and low-density diets from 1 to 38 days of age.

\begin{tabular}{|c|c|c|c|c|c|c|c|c|}
\hline \multicolumn{2}{|c|}{ Treatment } & $\begin{array}{c}\text { WBCs, } \\
10^{6} / \mathrm{mm}^{3}\end{array}$ & Lymphocytes, \% & Monocytes, \% & Basophils, \% & Eosinophils, \% & Heterophils, \% & H/L Ratio \\
\hline \multicolumn{9}{|c|}{ Effect of diet } \\
\hline \multicolumn{2}{|c|}{$\begin{array}{l}\text { Regular } \\
\text { density }\end{array}$} & 22.8 & $41.1^{\mathrm{b}}$ & 10.5 & 0.583 & $10.0^{\mathrm{a}}$ & 23.4 & 0.572 \\
\hline \multicolumn{2}{|c|}{ Low density } & 22.7 & $42.4^{\mathrm{a}}$ & 10.6 & 0.583 & $9.29^{b}$ & 23.7 & 0.564 \\
\hline \multicolumn{9}{|c|}{ Effect of yeast concentration } \\
\hline \multirow{3}{*}{\multicolumn{2}{|c|}{$\begin{array}{c}\text { Control } \\
0.02 \% \\
0.04 \%\end{array}$}} & 22.8 & $40.0^{\mathrm{b}}$ & $9.68^{b}$ & 0.562 & 9.87 & $23.1^{\mathrm{b}}$ & $0.583^{\mathrm{a}}$ \\
\hline & & 23.1 & $42.9^{\mathrm{a}}$ & $11.3^{\mathrm{a}}$ & 0.625 & 9.56 & $23.4^{\mathrm{b}}$ & $0.550^{\mathrm{b}}$ \\
\hline & & 22.4 & $42.4^{\mathrm{a}}$ & $10.8^{a}$ & 0.562 & 9.56 & $24.1^{a}$ & $0.571^{a, b}$ \\
\hline \multicolumn{9}{|c|}{ Interaction between diet and yeast concentration } \\
\hline Regular & Control & $21.7^{\mathrm{b}}$ & 39.7 & 9.25 & 0.625 & 10.6 & 23.0 & $0.585^{\mathrm{a}}$ \\
\hline den- & $0.02 \%$ & $23.2^{\mathrm{a}}$ & 41.7 & 11.5 & 0.750 & 9.87 & 23.7 & $0.571^{\mathrm{a}}$ \\
\hline & $0.04 \%$ & $23.5^{\mathrm{a}}$ & 42.0 & 11.0 & 0.375 & 9.62 & 23.5 & $0.560^{\mathrm{a}}$ \\
\hline Low & Control & $23.8^{a}$ & 40.3 & 10.1 & 0.500 & 9.12 & 23.2 & $0.581^{\mathrm{a}}$ \\
\hline den- & $0.02 \%$ & $23.0^{\mathrm{a}}$ & 44.1 & 11.1 & 0.500 & 9.25 & 23.1 & $0.528^{b}$ \\
\hline sity & $0.04 \%$ & $21.3^{b}$ & 42.8 & 10.6 & 0.750 & 9.50 & 24.8 & $0.583^{a}$ \\
\hline \multicolumn{2}{|c|}{ SEM } & 0.450 & 0.543 & 0.379 & 0.189 & 0.300 & 0.362 & 0.130 \\
\hline \multicolumn{9}{|c|}{$p$ value } \\
\hline & 0.8219 & 0.0059 & 0.8939 & 1.0000 & 0.0041 & 0.2689 & 0.4844 \\
\hline \multicolumn{2}{|c|}{ Yeast } & 0.3213 & 0.0001 & 0.0004 & 0.9304 & 0.4936 & 0.0174 & 0.0453 \\
\hline \multicolumn{2}{|c|}{ Interaction } & 0.0001 & 0.2324 & 0.1783 & 0.2321 & 0.0815 & 0.3219 & 0.0485 \\
\hline
\end{tabular}

a,b Means within a column with different superscripts are significantly different based on Student-Newman-Keuls (SNK) post hoc tests. $\mathrm{H} / \mathrm{L}=$ Heterophil to lymphocyte ratio. Number of observations was 6 per interaction cell.

There were significant effects of interaction between dietary treatments and SC concentrations on PCV, Hgb, MCV, MCH, white blood cells (WBCs) and the H/L ratio. Results showed that SC supplementation increased PCV and Hgb for birds fed a regular-density diet, but $0.4 \mathrm{~g} / \mathrm{kg}$ SC significantly decreased Hgb in birds fed a low-density diet. In addition, MCV and MCH were increased dramatically by the addition of $0.04 \%$ SC to the regular-density diet, but they were reduced in birds fed the low-density diet. The results showed that using $0.02 \%$ and $0.04 \%$ of SC increased WBCs in birds fed a regular-density diet, but $0.04 \%$ of SC significantly decreased WBCs in those fed a low-density diet. On the other hand, $0.02 \%$ of SC significantly decreased H/L in birds fed the low-density diet only (Table 5).

\subsection{Lymph Organs and Immune Response}

Table 6 shows the effects of the experimental treatments on the lymphoid organs of broilers. The results showed no significant effect of diet density on lymph structures like the spleen, absolute weight of thymus and bursa of Fabricius. However, the percentage of thymus was significantly greater in birds fed a low-density diet than that in birds fed a regular-density diet. Immune responses to NDV and AI, as measured by HI, were not influenced by diet type. These organs, as well as the immune response to NDV and AI, were significantly greater in broilers fed a diet supplemented with $0.02 \%$ or $0.04 \%$ of SC than those fed a diet without SC supplementation. Moreover, the effects on the thymus, bursa of Fabricius and NDV and AI showed stepwise increases. There were significant effects of interactions between diet type and SC on the percentage of spleen weight, bursa weight and immune response to NDV. The results indicated that the absolute weights of the spleen and bursa of Fabricius significantly decreased in the group fed the regular-density diet supplemented with $0.04 \%$ of SC. Still, both levels of SC significantly increased immune response to NDV. On the other hand, both concentrations of SC significantly increased the absolute weights of the spleen and bursa of Fabricius in birds fed the low-density diet, but the response to NDV was stepwise (Table 6). 
Table 6. Impact of different concentrations of Saccharomyces cerevisiae on immune organs and HI titer $(\log 2)$ in response to avian influenza and Newcastle disease virus in 38-day-old broilers fed either a regular- or low-density diet from days 1 to 38 of age.

\begin{tabular}{|c|c|c|c|c|c|c|}
\hline \multirow{2}{*}{\multicolumn{2}{|c|}{ Treatment }} & \multirow{2}{*}{ Spleen, \% } & \multirow{2}{*}{ Thymus, \% } & \multirow{2}{*}{$\begin{array}{c}\text { Bursa of } \\
\text { Fabricius, \% }\end{array}$} & \multicolumn{2}{|c|}{$\mathrm{HI}^{2}, \mathrm{Log} 2$} \\
\hline & & & & & NDV & AI \\
\hline \multirow{3}{*}{\multicolumn{2}{|c|}{$\begin{array}{l}\text { Regular density } \\
\text { Low density }\end{array}$}} & \multicolumn{3}{|c|}{ Effect of diet } & & \\
\hline & & 0.135 & $0.466^{\mathrm{b}}$ & 0.201 & 3.36 & 2.83 \\
\hline & & 0.148 & $0.516^{\mathrm{a}}$ & 0.223 & 3.43 & 2.04 \\
\hline \multicolumn{7}{|c|}{ Effect of yeast concentration } \\
\hline \multicolumn{2}{|c|}{ Control } & $0.110^{b}$ & $0.425^{\mathrm{c}}$ & $0.167^{\mathrm{c}}$ & $1.12^{\mathrm{c}}$ & $0.125^{c}$ \\
\hline \multicolumn{2}{|c|}{$0.02 \%$} & $0.153^{\mathrm{a}}$ & $0.490^{\mathrm{b}}$ & $0.213^{b}$ & $3.54^{\mathrm{b}}$ & $2.69^{b}$ \\
\hline \multicolumn{2}{|c|}{$0.04 \%$} & $0.161^{\mathrm{a}}$ & $0.558^{\mathrm{a}}$ & $0.256^{\mathrm{a}}$ & $5.58^{\mathrm{a}}$ & $4.50^{\mathrm{a}}$ \\
\hline \multicolumn{7}{|c|}{ Interaction between diet and yeast concentration } \\
\hline \multirow{3}{*}{$\begin{array}{l}\text { Regular } \\
\text { density }\end{array}$} & Control & $0.112^{\mathrm{c}}$ & $0.366^{\mathrm{c}}$ & $0.162^{b}$ & $1.17^{\mathrm{d}}$ & 0.250 \\
\hline & $0.02 \%$ & $0.136^{\mathrm{b}}$ & $0.486^{b}$ & $0.189^{b}$ & $3.92^{c}$ & 3.37 \\
\hline & $0.04 \%$ & $0.155^{\mathrm{a}, \mathrm{b}}$ & $0.545^{\mathrm{a}, \mathrm{b}}$ & $0.252^{a}$ & $5.00^{b}$ & 4.87 \\
\hline \multirow{3}{*}{$\begin{array}{c}\text { Low } \\
\text { density }\end{array}$} & Control & $0.108^{c}$ & $0.484^{b}$ & $0.171^{b}$ & $1.08^{\mathrm{d}}$ & 0.00 \\
\hline & $0.02 \%$ & $0.169^{\mathrm{a}}$ & $0.495^{b}$ & $0.238^{a}$ & $3.17^{c}$ & 2.00 \\
\hline & $0.04 \%$ & $0.167^{\mathrm{a}}$ & $0.570^{\mathrm{a}}$ & $0.260^{\mathrm{a}}$ & $6.17^{\mathrm{a}}$ & 4.12 \\
\hline \multicolumn{2}{|l|}{ SEM } & 0.326 & 0.195 & 0.014 & 0.372 & 0.605 \\
\hline \multirow{2}{*}{\multicolumn{2}{|c|}{ Diet }} & & $p$ value & & & \\
\hline & & 0.0669 & 0.0214 & 0.0606 & 0.7161 & 0.1174 \\
\hline \multicolumn{2}{|c|}{ Yeast } & 0.0001 & 0.0001 & 0.0001 & 0.0001 & 0.0001 \\
\hline \multicolumn{2}{|c|}{ Interaction } & 0.1060 & 0.0828 & 0.2739 & 0.0393 & 0.6515 \\
\hline
\end{tabular}

$\overline{\mathrm{a}, \mathrm{b}, \mathrm{c}, \mathrm{d}}$ Means within a column with different superscripts are significantly different based on Student-NewmanKeuls (SNK) post hoc tests. $\mathrm{HI}=$ Hemagglutination inhibition test; NDV = Newcastle disease virus; I = Influenza antigen. Number of observations was 6 chicks per interaction cell.

\subsection{Immune Indices}

Tables 7 and 8 show the impacts of the different experimental treatments on the immune parameters and survival rate of broilers, respectively. Diet type significantly influenced LYS, TAC and PA, revealing an increasing effect of a low-density diet on immune parameters, but lower TAC (Table 7).

Supplementation of the diet with SC significantly decreased TAC, but enhanced PI relative to the standard diet and had no impact on other traits related to the immunity, including serum LTT, BACT, LYS and PA (Table 7). In addition, the survival rate of broiler chickens fed a diet fortified with $0.04 \%$ of SC was significantly greater than that of chickens fed a dietary supplement of $0.02 \%$ SC, or a diet without SC (Table 8 ).

The interaction between SC concentration and diet type did not influence the LTT, BACT, LYS, TAC, PI, PA, or survival rate during the challenge experiment (Tables 7 and 8).

Table 7. Impact of different concentrations of Saccharomyces cerevisiae on immune indices of broilers fed a regular- or low-density diet from 1 to 38 days of age.

\begin{tabular}{ccccccc}
\hline Treatment & LTT, $\%$ & BACT, $\%$ & LYS, $\%$ & $\begin{array}{c}\text { TAC, } \\
\text { mMol/dL }\end{array}$ & PI & PA, \% \\
\hline Regular density & 24.2 & 42.1 & $0.082^{\mathrm{b}}$ & $429^{\mathrm{a}}$ & 1.58 & $17.4^{\mathrm{b}}$ \\
Low density & 25.8 & 43.2 & $0.098^{\mathrm{a}}$ & $419^{\mathrm{b}}$ & 1.53 & $18.8^{\mathrm{a}}$ \\
SEM & 0.599 & 0.527 & 0.004 & 2.19 & 0.025 & 0.375 \\
\hline \multicolumn{7}{c}{ Effect of diet } \\
Control & 24.8 & 43.9 & 0.081 & $436^{\mathrm{a}}$ & $1.46^{\mathrm{b}}$ & 17.5 \\
$0.02 \%$ & 25.1 & 41.8 & 0.095 & $420^{\mathrm{b}}$ & $1.61^{\mathrm{a}}$ & 18.3 \\
$0.04 \%$ & 25.0 & 42.3 & 0.095 & $416^{\mathrm{b}}$ & $1.60^{\mathrm{a}}$ & 18.6 \\
SEM & 0.733 & 0.645 & 0.005 & 2.68 & 0.031 & 0.460 \\
\hline
\end{tabular}


Table 7. Cont.

\begin{tabular}{|c|c|c|c|c|c|c|c|}
\hline \multicolumn{2}{|c|}{ Treatment } & LTT, \% & ВАCТ, $\%$ & LYS, \% & $\begin{array}{c}\text { TAC, } \\
\mathrm{mMol} / \mathrm{dL}\end{array}$ & PI & $\mathbf{P A}, \%$ \\
\hline \multicolumn{8}{|c|}{ Interaction between diet and yeast concentration } \\
\hline \multirow{3}{*}{$\begin{array}{l}\text { Regular } \\
\text { density }\end{array}$} & Control & 23.6 & 43.2 & 0.071 & 443 & 1.45 & 16.0 \\
\hline & $0.02 \%$ & 24.3 & 41.2 & 0.082 & 425 & 1.61 & 17.8 \\
\hline & $0.04 \%$ & 24.7 & 42.0 & 0.092 & 419 & 1.68 & 18.5 \\
\hline \multirow{3}{*}{$\begin{array}{c}\text { Low } \\
\text { density }\end{array}$} & Control & 26.1 & 44.6 & 0.091 & 430 & 1.47 & 19.0 \\
\hline & $0.02 \%$ & 26.0 & 42.5 & 0.107 & 414 & 1.61 & 18.8 \\
\hline & $0.04 \%$ & 25.3 & 42.7 & 0.097 & 413 & 1.52 & 18.7 \\
\hline \multicolumn{2}{|c|}{ SEM } & 1.03 & 0.913 & 0.007 & 3.39 & 0.044 & 0.650 \\
\hline \multicolumn{8}{|c|}{$p$-value } \\
\hline \multicolumn{2}{|c|}{ Diet } & 0.0693 & 0.1394 & 0.0128 & 0.0027 & 0.2240 & 0.0111 \\
\hline \multicolumn{2}{|c|}{ Yeast } & 0.9552 & 0.0746 & 0.1410 & 0.0001 & 0.0026 & 0.2054 \\
\hline \multicolumn{2}{|c|}{ Interaction } & 0.6676 & 0.9366 & 0.4206 & 0.6813 & 0.0942 & 0.1052 \\
\hline
\end{tabular}

$\overline{\mathrm{a}, \mathrm{b}}$ Means within a column with different superscripts are significantly different based on Student-Newman-Keuls (SNK) post hoc tests. $\mathrm{LTT}=$ Lymphocyte transformation test; $\mathrm{BACT}=$ Bactericidal activity; $\mathrm{LYS}=$ Lysozyme activity; TAC = Total antioxidant capacity; PI = Phagocytic index; PA = Phagocytic activity. Number of observations was 6 per interaction cell.

Table 8. Impact of different concentrations of Sacharomyces cerevisiae on the survival rate of broiler chickens between 38 and 48 days of age that were fed a regular- or low-density diet and infected with HPAIV H5N1 at 38 days of age.

\begin{tabular}{|c|c|c|}
\hline \multicolumn{2}{|c|}{ Treatment } & $\begin{array}{c}\text { Survival Rate after Infection } \\
\text { of Influenza at } 38 \text { of Age, } \%\end{array}$ \\
\hline \multicolumn{3}{|c|}{ Effect of diet } \\
\hline \multirow{2}{*}{\multicolumn{2}{|c|}{$\begin{array}{l}\text { Regular density } \\
\text { Low density }\end{array}$}} & 64.8 \\
\hline & & 59.2 \\
\hline \multicolumn{3}{|c|}{ Effect of yeast concentration } \\
\hline \multicolumn{2}{|c|}{ Control } & $56.0^{\mathrm{b}}$ \\
\hline \multicolumn{2}{|c|}{$0.02 \%$} & $56.0^{\mathrm{b}}$ \\
\hline \multicolumn{2}{|c|}{$0.04 \%$} & $71.0^{\mathrm{a}}$ \\
\hline \multicolumn{3}{|c|}{ Effect of age } \\
\hline & $100^{\mathrm{a}}$ \\
\hline \multicolumn{2}{|c|}{$40 \mathrm{~d}(2)$ challenge } & $100^{\mathrm{a}}$ \\
\hline \multicolumn{2}{|c|}{$41 \mathrm{~d}(3)$ challenge } & $70.0^{\mathrm{b}}$ \\
\hline \multicolumn{2}{|c|}{$42 \mathrm{~d}(4)$ challenge } & $56.6^{\mathrm{b}, \mathrm{c}}$ \\
\hline \multicolumn{2}{|c|}{$43 \mathrm{~d}(5)$ challenge } & $50.0^{b, c}$ \\
\hline \multicolumn{2}{|c|}{$44 \mathrm{~d}(6)$ challenge } & $50.0^{b, c}$ \\
\hline \multicolumn{2}{|c|}{$45 \mathrm{~d}(7)$ challenge } & $50.0^{b, c}$ \\
\hline \multicolumn{2}{|c|}{$46 \mathrm{~d}(8)$ challenge } & $45.0^{\mathrm{b}, \mathrm{c}}$ \\
\hline \multicolumn{2}{|c|}{47 d (9) challenge } & $40.0^{\mathrm{c}}$ \\
\hline \multicolumn{2}{|c|}{$48 \mathrm{~d}(10)$ challenge } & $30.0^{\mathrm{c}}$ \\
\hline \multicolumn{3}{|c|}{ Interaction between diet and yeast concentration } \\
\hline \multirow{4}{*}{ Regular density } & Control & 56.0 \\
\hline & $0.02 \%$ & 64.0 \\
\hline & $0.04 \%$ & 70.0 \\
\hline & Control & 56.0 \\
\hline \multirow[t]{3}{*}{ Low density } & $0.02 \%$ & 48.0 \\
\hline & $0.04 \%$ & 72.0 \\
\hline & & 6.57 \\
\hline & $p$ value & \\
\hline & & 0.2947 \\
\hline & & 0.0001 \\
\hline & & 0.1380 \\
\hline
\end{tabular}

$\overline{a, b, c}$ Means within a column with different superscripts are significantly different based on Student-NewmanKeuls (SNK) test. Number of observations was 6 broilers of 38-day-old per interaction cell. 


\subsection{Histology Study}

Table 9 shows the impact of diet type and SC concentration on the morphology of the intestines and bursa of Fabricius and spleen, as shown in Figures 1-3. Diet type did not influence the length of intestinal villi or diameter of the large follicle of the bursa of Fabricius (Table 9). No changes were seen in the spleen and thymus due to diet type, SC concentration, or the interaction between these terms (Table 9; Figures 2 and 3).

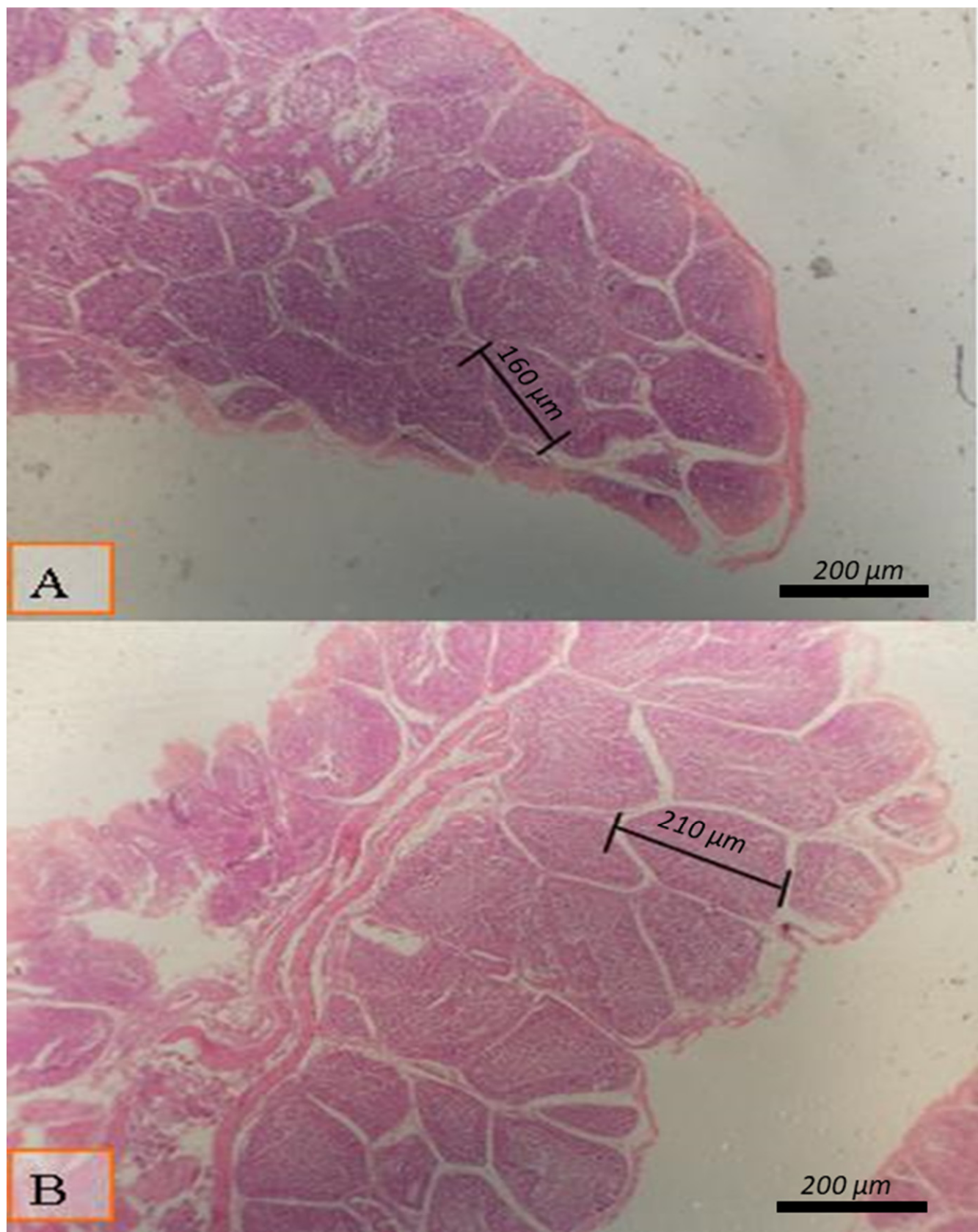

Figure 1. Micrograph of bursa of Fabricious of broiler at day 38 of age stained with HandE (X40) to investigate the follicle diameter in different groups; the distance between two follicular polar as presented all groups by lines: (A) Broilers supplemented with $0.02 \%$ Saccharomyces cerevisiae;(B) broilers supplemented with $0.04 \%$ Saccharomyces cerevisiae. Moderate enhancement in the follicular diameter was detected in broilers supplemented with $0.04 \%$ Saccharomyces cerevisiae (B). 
Table 9. Impact of different concentrations of Saccharomyces cerevisiae on the morphology of the intestine, bursa of Fabricius and follicular cortical:medullary ratio of the thymus in 38-day-old broilers fed regular- or low-density diet.

\begin{tabular}{|c|c|c|c|c|}
\hline \multicolumn{2}{|c|}{ Treatment } & $\begin{array}{c}\text { Length of Intestinal } \\
\text { Villi, } \mu \mathrm{m}\end{array}$ & $\begin{array}{l}\text { L. Axis of Large } \\
\text { Follicle of Bursa } \\
\text { of Fabricius, } \mu \mathrm{m}\end{array}$ & $\begin{array}{l}\text { Follicular Cortical: } \\
\text { Medullary Ratio of } \\
\text { the Thymus }\end{array}$ \\
\hline \multicolumn{5}{|c|}{ Effect of diet } \\
\hline \multicolumn{2}{|c|}{ Regular density } & 239 & 166 & $1: 3$ \\
\hline \multicolumn{2}{|c|}{ Low density } & 248 & 177 & $1: 3$ \\
\hline \multicolumn{5}{|c|}{ Effect of yeast concentration } \\
\hline \multicolumn{2}{|c|}{ Control } & $215^{\mathrm{b}}$ & $157^{\mathrm{b}}$ & $1: 3$ \\
\hline \multicolumn{2}{|c|}{$0.02 \%$} & $227^{b}$ & $164^{\mathrm{b}}$ & 1.3 \\
\hline \multicolumn{2}{|c|}{$0.04 \%$} & $289^{a}$ & $193^{\mathrm{a}}$ & $1: 3$ \\
\hline \multicolumn{5}{|c|}{ Interaction between diet and yeast concentration } \\
\hline \multirow{3}{*}{$\begin{array}{l}\text { Regular } \\
\text { density }\end{array}$} & Control & 211 & 157 & $1: 3$ \\
\hline & $0.02 \%$ & 226 & 159 & $1: 3$ \\
\hline & $0.04 \%$ & 279 & 181 & $1: 3$ \\
\hline \multirow{3}{*}{$\begin{array}{l}\text { Low } \\
\text { density }\end{array}$} & Control & 219 & 157 & $1: 3$ \\
\hline & $0.02 \%$ & 227 & 170 & $1: 3$ \\
\hline & $0.04 \%$ & 298 & 206 & $1: 3$ \\
\hline \multicolumn{2}{|c|}{ SEM } & 9.61 & 8.07 & ND \\
\hline \multicolumn{5}{|c|}{$p$ value } \\
\hline \multicolumn{2}{|c|}{ Diet } & 0.2389 & 0.0851 & ND \\
\hline \multicolumn{2}{|c|}{ Yeast } & 0.0001 & 0.0002 & ND \\
\hline \multicolumn{2}{|c|}{ Interaction } & 0.6707 & 0.2832 & ND \\
\hline
\end{tabular}

$\overline{\mathrm{a}, \mathrm{b}}$ Means within a column with different superscripts are significantly different based on Student-Newman-Keuls (SNK) post hoc tests. Number of observations was 6 per interaction cell per age. ND $=$ Not done.

Supplementation of the diet with $0.04 \%$ SC significantly enhanced the length of intestinal villi (Table 9) and the diameter of the large bursal follicle (Table 9 and Figure 1). The intestinal villi and bursa of Fabricious were enhanced by $29.8 \%$ and $22.9 \%$, respectively. Both traits were significantly enhanced with the supplementation of SC at a concentration of $0.02 \%$. The large bursal follicle's intestinal villi length and diameter were not affected by the interaction between diet type and SC concentration.

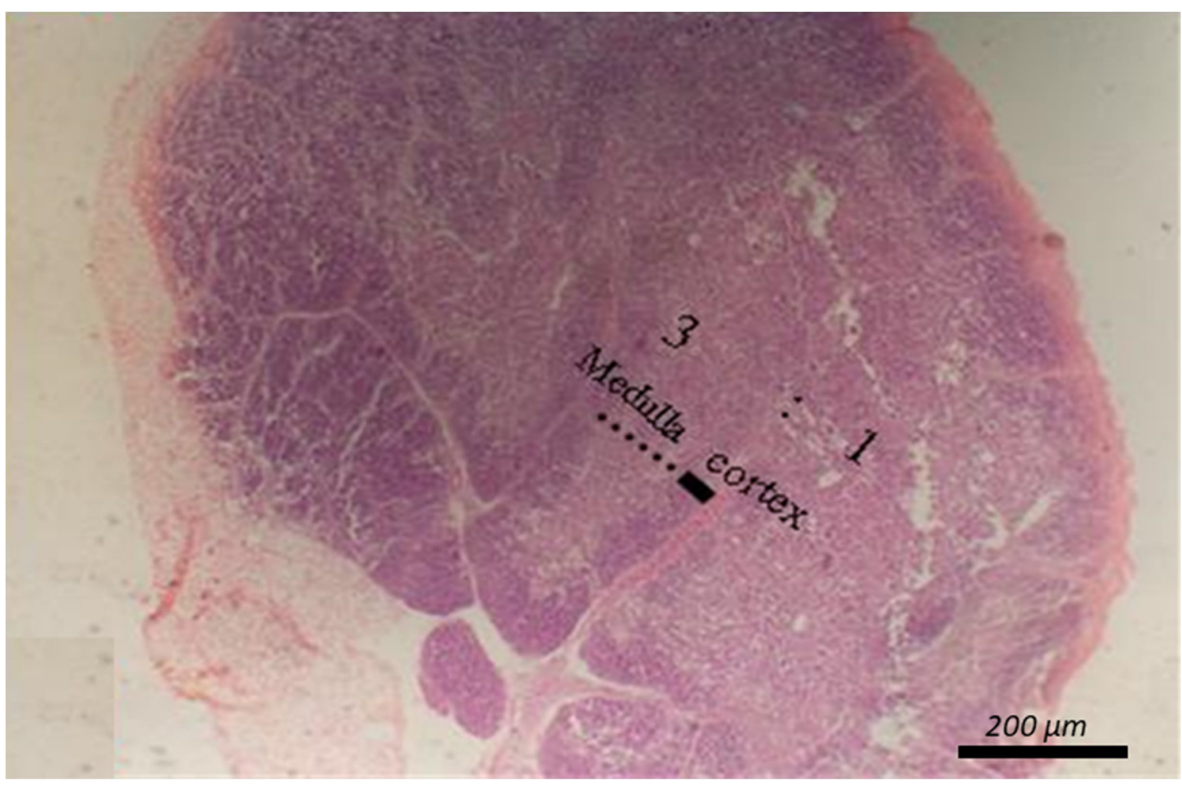

Figure 2. Micrograph of the thymus stained with HandE (X40) to explore the thymic cortical: medullary ratio. 


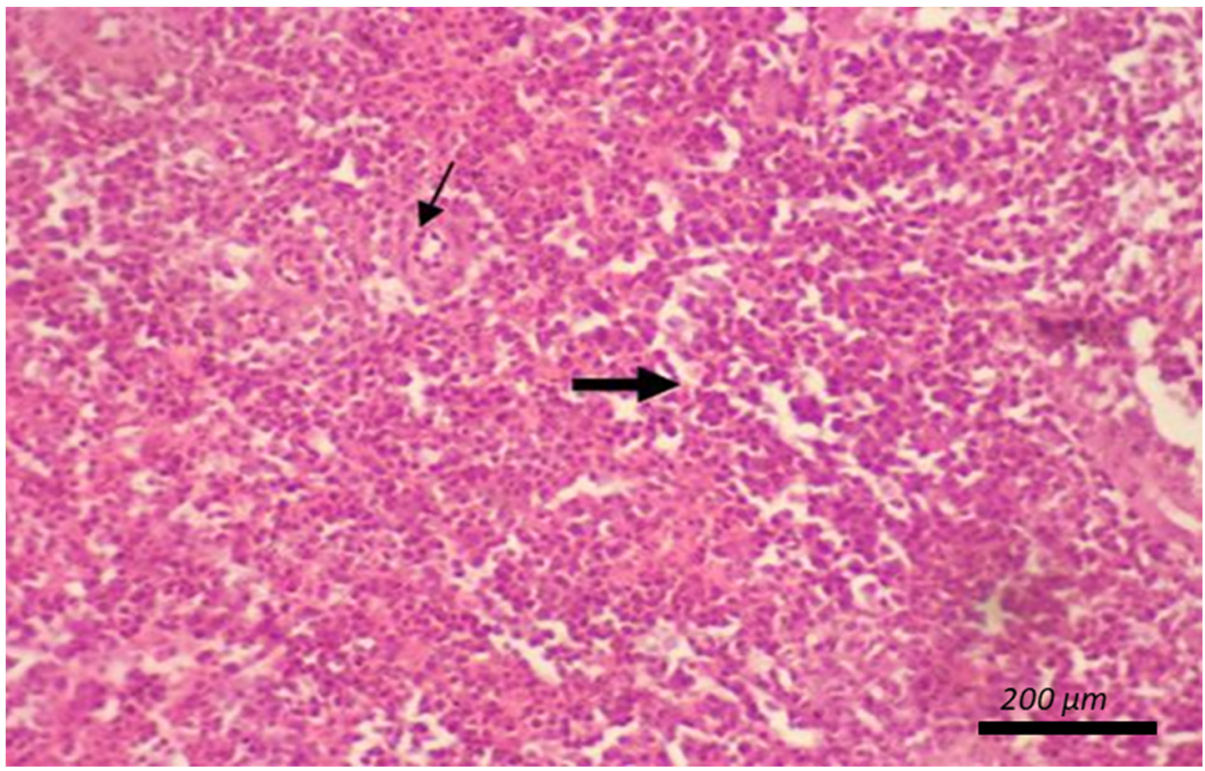

Figure 3. Micrograph of the spleen stained with HandE (X40) of regular density diet presented normal splenic histology featuring splenic arteriole (thin arrow) with white and red pulp (thick arrow). All groups presented the normal splenic histology as the control.

\section{Discussion}

The present work was conducted to fill the gap in knowledge regarding the impact of active SC yeast in relation to dietary composition on the antioxidant status, blood constituents and immune response of broiler chickens. Adding an SC product to the feed at concentrations of $0.02 \%$ or $0.04 \%$ improved the performance of broilers from 1 to 38 days of age.

To our knowledge, this is the first study investigating the interactive relationship between using yeast in low-density diets and its effect on immune response in broiler chickens. The percentage of thymus was significantly greater in birds fed a low-density diet than that of birds fed a regular-density diet. The immune response to NDV and AI were significantly greater in broilers fed a diet supplemented with $0.02 \%$ or $0.04 \%$ of SC than those fed a diet without SC supplementation. In addition, there were significant interactions between diet type and SC on the percentage of spleen weight, bursa weight and immune response to NDV. The results indicated that the absolute weights of the spleen and bursa of Fabricious were significantly high in the group fed the low-density diet supplemented with $0.04 \%$ of SC. Still, both levels of SC significantly increased immune response to NDV. Interestingly, both concentrations of SC significantly increased the absolute weights of the spleen and bursa of Fabricious in birds fed the low-density diet.

The increase in feed cost accompanied by the reduction in the availability of corn as a main feed ingredient will affect the production efficiency of poultry on the global level, especially during global pandemics such as the current coronavirus crisis. Nutritional manipulation by using the low-density diet supplemented with yeast could provide a great opportunity to improve the economic outcome by reducing the feed cost, which constitutes approximately $60-70 \%$ of the total poultry operation cost. Using low densitydiet in broiler rations will provide a positive alternative to reduce feed cost. The other side of the coin is that using yeast in these diets compensated for the low-density contents of the diets by improving the antioxidant status and immune response of the broiler chickens. Any improvement in nutrition management and feed cost will have a direct impact on profitability and efficiency of poultry industry $[49,50]$.

In addition, the group treated with $0.04 \%$ of SC had a significantly higher immune response than the other groups. These results contribute to the poultry industry important information that will improve production efficiency. 
In addition, $0.02 \%$ of $\mathrm{SC}$ decreased MAD of broilers fed a low-density diet compared with a regular-density diet. These results indicate that SC enhances the oxidative status of broilers. Interestingly, Czech et al. [11] revealed that using 3\% of Yarrowia lipolytica or SC yeast, in combination with Bacillus sp. probiotic, in the feed of turkeys from 7 to 112 days of age improved the antioxidant status of birds by preventing lipid peroxidation. This effect enhances the ability of poultry to handle stress and infectious agents. In another study [10], the authors investigated the mechanism by which SC enhances the oxidative status of broiler chickens. The authors included SC in either the feed or drinking water of stressed broilers and measured CYP1A2 and melanocortin-2 receptor (MC2R) gene expression in the adrenal glands and IL10 and AvBD1 in the spleen. The authors concluded that using SC in broilers' feed or drinking water for 40 days decreased stress and MC2R gene expression. They also showed that supplementation of SC fermentate in the feed was marginally more effective than adding it to drinking water in stimulating oxidative status and reducing stress in broiler chickens [10] and in detoxifying nitrate $(21,22)$ and aflatoxin $(22,23)$.

There has been an interest in using a low-density diet to feed broilers, to lower the growing pressure on the skeletal system of the bird and decrease skeletal diseases, the cost of feed and environmental pollution [51,52].

The body weight and EPEI were significantly decreased by $7.8 \%$ and $5.4 \%$, respectively, over the study period for birds on the low-density diet compared to the regular-density diet. Indeed, the main effect of regular diet under the three SC levels was $2099 \mathrm{~g}$ while the mean body weight of the control diet without SC supplementations $(1946+1784)$ was $1965 \mathrm{~g}$. These findings indicate that the negative impact of diet structure persisted during the experimental period from 1 to 38 days of age [53].

The outcomes showed that the low-density diet improved liver function and increased the percentage of thymus and lymphocytes and PA, but decreased $\gamma$-globulin, eosinophils, TAC, ALT and AST. The current study revealed that supplementation of the feed with SC at $0.02 \mathrm{~g} / \mathrm{kg}$ and $0.04 \%$ significantly lowered serum AST, ALT and MAD relative to the standard diet. Gheisari and Kholeghipour [9] showed that using live yeast had no significant impact on hematological indices such as RBCs, WBCs and PCV. On the other hand, in another study, there was a positive association between supplementation of feed with SC and hematological traits of chickens, such as RBCs, WBCs and PCV [51]. In the same study, probiotics had no effect $(p>0.05)$ on hemoglobin and WBCs at the finisher phase. Yet, a significant effect $(p<0.05)$ was observed for RBCs and packed cell volume.

Gut morphology was modulated because of the addition of $10 \%$ wheat bran in the lowdensity diet. The results indicated that the diets had no impact on the length of intestinal villi. Previous studies have shown that dietary cereal with a high nonstarch polysaccharides (NSP) level could enhance the dimension of the gastrointestinal tract [54]. Steenfeldt [55] observed that the arabinoxylan level in wheat is significantly and positively correlated to the relative masses of the duodenum, jejunum and ileum. It has been stated that dietary supplementation may modulate the morphology of the intestinal mucosa. Accordingly, NSP in the diet can also impact the morphology of the gastrointestinal tract [56]. Iji [57] showed that the crypt deepness of the jejunum and ileum was significantly enhanced by dietary addition of guar gum and xanthin gum. This finding demonstrated that NSP can improve cell turnover in the gastrointestinal tract. Enhanced crypt deepness indicates enhanced villus cell proliferation and in turn improved utilization of the nutrients by the gastrointestinal tract. This suggests that these cereals impact the mass of the gastrointestinaltract and morphology of the intestine [58].

These results suggest that the dilution of nutrients in feed via adding $10 \%$ wheat bran improves the immune response and production index of broilers 1 to 38 days of age. These outcomes agree with earlier studies by Abudabos [53] and Attia [54]. Wheat bran polysaccharides have been shown to act as antioxidants and immunostimulators and have anti-inflammatory, antitussive, anticancerous and antimutagenic properties [59-62]. Furthermore, wheat bran arabinoxylans have been shown to to enhance macrophage phagocytosis in animals [63]. They are immunostimulants of the antibody response in 
chickens by enhancing the total IgG and IgM anti-SRBC antibody titers on 7th and 14th day post primary antibody response (PPI) and post-secondary inoculation (PSI) of sheep red blood cells (SRBCs) compared to the control. Additionally, Korte et al. [64] stated that supplementing feed with arabinoxylans significantly induced anti-SRBC antibody titers, representing enhanced humoral immunity in chickens.

The results indicated that supplementing feed with SC at either $0.02 \%$ or $0.04 \%$ significantly affected growth and EPEI relative to the control dietary treatment. The effect persisted throughout all tested periods. Furthermore, supplementing the low-density diet with $0.02 \%$ or $0.04 \%$ of SC resulted in an enhanced production index compared with the regular-density diet lacking the addition.

These results agree with other studies that have investigated similar effects of SC on growth performance [65-68]. In addition, the positive effect of a higher dose of SC are in line with results reported by Valdivie [69], who found that the growth performance of broilers significantly improved with an increased supplementation dose of SC. The improved gastrointestinal health and growth performance of broilers supplemented with SC may be due to the presence of effective ingredients in SC such as Vitamin B, cellulostic enzymes, phytase, monooligosaccharides (MOS) and glucomannan [70].

A diet supplemented with $0.04 \%$ SC increased the length of the villi and SC supplementation enhanced the production index and the body weight of broiler from 1 to 38 days of age in a dose-dependent manner.

There were significant improvements in blood serum biochemistry and liver function due to SC supplementation. Consistent with these results, Paryad and Mahmoudi [71] and Hosseini [72] showed that SC at 1.5\% significantly enhanced total plasma protein, albumin and globulin and WBCs and decreased the H/L ratio. Furthermore, Zhang et al. [64] revealed that SC supplementation to broiler chickens significantly lowered the 2-thiobarbituric acid-reactive substances (TBARS) in the breast and drumstick meats and increased villus height, compared to the control group.

Supplementation of SC significantly enhanced the spleen, thymus, bursa of Fabricious and $\mathrm{HI}$ in response to NDV and AI, with a positive concentration-dependent impact of SC on the thymus, bursa of Fabricious and immune response to NDV and AI. The diameter of the bursal follicle significantly enhanced at $0.04 \%$ SC, indicating an improvement in the number of B-lymphoblasts, leading to an increase in the B-lymphocytes responsible of humoral immunity stimulation through antibody production. Further evidence of this effect was reflected by the increased survival rate of broilers challenged with AI at $38 \mathrm{~d}$ of age. In addition, SC supplementation was associated with improvements in $\beta$-globulin and hematological traits such as PCV, Hgb, RBCs, lymphocytes, monocytes and PI. These data provide more evidence for an improved health status of broilers fed a diet supplemented with SC. The effect of SC on the relative weights of the thymus and bursa and immune response to NDV and AI, was dose-dependent. Similarly, Newman [73], Spring et al. [74] and Zhang et al. [70] showed that SC supplementation of the diet enhanced production performance by improving the immune status, intestinal lumen health and digestion and nutrient utilization of birds. In addition, Gheisari and Kholeghipour [9] found that broiler chickens fed SC at a concentration of $0.02 \%$ had higher antibody titers against NDV than the control at $38 \mathrm{~d}$ of age, but it did not affect AI titers. The positive effect of SC on immune response could be attributed to its cell wall constituents, including chitin, mannan and glucan, which have immunostimulant effects [2,71,75-78].

\section{Conclusions}

The antioxidant status and total antioxidant capacity of broiler chickens were improved by supplementation of the diet with SC. Supplementation of either a regular-density diet or a low-density diet with SC at either $0.02 \%$ or $0.04 \%$ enhanced the BWG and EPEI of broilers during 1 to 38 days of age. Additionally, broilers fed a low-density diet supplemented with either $0.02 \%$ or $0.04 \%$ SC had a greater body weight and EPEI than birds fed the control 
diet with no supplementary SC. However, fortification of the diet with $0.04 \%$ resulted in significantly enhanced immune organs and a higher immune response.

Author Contributions: Y.A.A., H.S.A.E.-H. and A.A.E.-S. conducted the experimental design and first draft of the manuscript; H.S.A.E.-H. and A.A.E.-S. carried out the experiment, data collection and contribution to the experimental set-up and laboratory work; H.A.-K. and S.R.A. carried out the statistical analyses and revision; Y.A.A., M.A.A.-H. and H.S.A.E.-H. proofread the manuscript. All authors have read and agreed to the published version of the manuscript.

Funding: The authors would like to thank the Kuwait Institute for Scientific Research, Kuwait, for providing the publications fees (APC).

Institutional Review Board Statement: The Animal Care and Use Committee (ACUC) at King Fahd Medical Research Center has approved the referenced protocol used in the current study, numbered ACUC-22-1-2, dated 1-2-2022.

Informed Consent Statement: Not applicable.

Data Availability Statement: Data were presented in the manuscript.

Acknowledgments: The Deanship of Scientific Research (DSR) at King Abdulaziz University, Jeddah, Saudi Arabia, has funded this project, under grant no. (FP-217-43H). The authors acknowledged the administrative, technical and financial support by DSR, King Abdulaziz University, Jeddah, Saudi Arabia.

Conflicts of Interest: The authors declare no conflict of interest.

\section{References}

1. Wang, W.; Li, Z.; Han, Q.; Guo, Y.; Zhang, B.; D'Inca, R. Dietary live yeast and mannan-oligosaccharide supplementation attenuate intestinal inflammation and barrier dysfunction induced by Escherichia coli in broilers. Br. J. Nutr. 2016, 116, 1878-1888. [CrossRef] [PubMed]

2. Attia, Y.A.; Al-Khalaifah, H.; Ibrahim, M.S.; Al-Hamid, A.E.A.; Al-Harthi, M.A.; El-Naggar, A. Blood Hematological and Biochemical Constituents, Antioxidant Enzymes, Immunity and Lymphoid Organs of Broiler Chicks Supplemented with Propolis, Bee Pollen and Mannan Oligosaccharides Continuously or Intermittently. Poult. Sci. 2017, 96, 4182-4192. [CrossRef] [PubMed]

3. Al-Khalifa, H.; Givens, D.I.; Rymer, C.; Yaqoob, P.; Juniper, D. The effect of increasing levels of Fish Oil on Immune Responses of Broiler Chickens. Advances in Animal Bio-Sciences Journal. Proc. Br. Soc. Anim. Sci. Annu. Conf. 2009, 2009, 235. [CrossRef]

4. Al-Khalifa, H.; Givens, D.I.; Rymer, C.; Yaqoob, P. Effect of n-3 fatty acids on immune function in broiler chickens. Poult. Sci. 2012, 91, 74-88. [CrossRef]

5. Al-Khalifa, H.; Ragheb, G.; Al-Nasser, A.; Al-Bahouh, M.; Khalil, F. Immunomodulation of Black Seed in Two Strains of Laying Hens. Int. J. Poult. Sci. 2013, 12, 451-455. [CrossRef]

6. Attia, Y.; Al-khalaifah, H.S.; Abd El-Hamid, H.; Al-Harthi, M.; El-Shafey, A. Multi-enzymes and immune response Effect of different levels of multi-enzymes on immune response, blood hematology and biochemistry, antioxidants status and organs histology of broiler chicks fed standard and low-density diets. Front. Vet. Sci. 2019, 6, 510. [CrossRef]

7. Flees, J.; Greene, E.; Ganguly, B.; Dridi, S.J.N. Phytogenic feed- and water-additives improve feed efficiency in broilers via modulation of (an)orexigenic hypothalamic neuropeptide expression. Neuropeptides 2020, 81, 102005. [CrossRef]

8. Suresh, G.; Santos, D.U.; Rouissi, T.; Hegde, K.; Brar, S.K.; Mehdi, Y.; Godbout, S.; Chorfi, Y.; Ramirez, A.A.J.A.F.S. Technology, In-field poultry tests to evaluate efficacy of bioformulation consisting of enzymes and yeast biomass. Anim. Feed. Sci. Technol. 2020, 262, 114398. [CrossRef]

9. Gheisari, A.A.; Kholeghipour, B. Effect of Dietary Inclusion of Live Yeast (Saccharomyces cerevisiae) on Growth Performance, Immune Responses and Blood Parameters of Broiler Chickens; World's Poultry Science Association (WPSA): Beekbergen, The Netherlands, 2006; p. 10.

10. Nelson, J.R.; Sobotik, E.B.; Athrey, G.; Archer, G.S.J.P.S. Effects of supplementing yeast fermentate in the feed or drinking water on stress susceptibility, plasma chemistry, cytokine levels, antioxidant status, and stress- and immune-related gene expression of broiler chickens. Poult. Sci. 2020, 99, 3312-3318. [CrossRef]

11. Czech, A.; Merska-Kazanowska, M.; Całyniuk, Z. Redox Status, Biochemical Parameters and Mineral Elements Content in Blood of Turkey Hens Fed a Diet Supplemented with Yarrowia lipolytica Yeast and Two Bacillus Species. Animals 2020, 10, 459. [CrossRef]

12. Leung, H.; Patterson, R.; Barta, J.; Karrow, N.; Kiarie, E. Nucleotide-rich yeast extract fed to broiler chickens challenged with Eimeria: Impact on growth performance, jejunal histomorphology, immune system, and apparent retention of dietary components and caloric efficiency. Poult. Sci. 2019, 98, 4375-4383. [CrossRef] [PubMed] 
13. Kiarie, E.G.; Leung, H.; Kakhki, R.A.M.; Patterson, R.; Barta, J.R. Utility of Feed Enzymes and Yeast Derivatives in Ameliorating Deleterious Effects of Coccidiosis on Intestinal Health and Function in Broiler Chickens. Front. Veter. Sci. 2019, 6, 473. [CrossRef] [PubMed]

14. Zhou, C.; Liang, J.; Jiang, W.; He, X.; Liu, S.; Wei, P. The effect of a selected yeast fraction on the prevention of pullorum disease and fowl typhoid in commercial breeder chickens. Poult. Sci. 2020, 99, 101-110. [CrossRef] [PubMed]

15. Mehdi, A.; Hasan, G. Immune response of broiler chicks fed yeast derived mannan oligosaccharides and humate against Newcastle disease. WASJ 2012, 18, 779-785.

16. Al-Khalifa, H. Production of added-value poultry meat: Enrichment with n-3 polyunsaturated fatty acids. World's Poult. Sci. J. 2015, 71, 319-326. [CrossRef]

17. Al-Khalifa, H.; Al-Nasser, A.; Al-Bahouh, M.; Ragheb, G.; Al-Qalaf, S.; Al-Omani, N.; Ahmad, A. The effect of polyunsaturated fatty acids on avian immune cell subpopulations in peripheral blood, spleen, and thymus. World's Poult. Sci. J. 2016, 72, 531-534. [CrossRef]

18. Al-Khalaifah, H.; Al-Nasser, A.; Givens, D.; Rymer, C.; Yaqoob, P. Comparison of different dietary sources of n-3 polyunsaturated fatty acids on immune response in broiler chickens. Heliyon 2020, 6, e03326. [CrossRef]

19. Al-Nasser, A.; Al-Khalaifah, H.; Al-Mansour, H.; Ahmad, A.; Ragheb, G. Evaluating farm size and technology use in poultry production in Kuwait. World's Poult. Sci. J. 2020, 76, 365-380. [CrossRef]

20. Attia, Y.A.; Abd El Hamid, A.E.; Ismaiel, A.M.; El-Naggar, A.S. The detoxication of nitrate by two antioxidants or a probiotic and the effects on blood and seminal plasma profiles and reproductive function of NZW rabbit bucks. Animal 2013, 7, 591-601. [CrossRef]

21. Attia, Y.A.; Abd El Hamid, A.E.; Ismaiel, A.M.; de Oliveira Maria, C.; Al-Harthi, M.A.; El-Naggar, A.S.; Simon, G.A. Nitrate detoxification using antioxidants and probiotics in the water of rabbits. Rev. Colomb. Cienc. Pecu. (RCCP) 2018, 31, 130-138. [CrossRef]

22. Attia, Y.; Al-Hamid, A.A.; Allakany, H.; Al-Harthi, M.; Mohamed, N. Necessity of continuing of supplementation of non-nutritive feed additive during days 21-42 of age following 3 weeks of feeding aflatoxin to broiler chickens. J. Appl. Anim. Res. 2015, 44, 87-98. [CrossRef]

23. Attia, Y.A.; Allakany, H.F.; Abd Al-Hamid, A.E.; Al-Saffar, A.A.; Hassan, R.A.; Mohamed, N.A. Capability of different nonnutritive feed additives on improving productive and physiological traits of broiler chicks fed diets with or without aflatoxin during the first 3 weeks of life. J. Anim. Physiol. Anim. Nutr. 2013, 97, 754-772. [CrossRef] [PubMed]

24. Hayat, J.; Savage, T.F.; Mirosh, L.W. The reproductive performance of two genetically distinct lines of medium white turkey hens when fed breeder diets with and without a yeast culture containing Saccharomyces cerevisiae. Anim. Feed. Sci. Technol. 1993, 43, 291-301. [CrossRef]

25. Arbor Acres Broiler Management Handbook. Available online: https://eu.aviagen.com/assets/Tech_Center/AA_Broiler/AABroilerHandbook2018-EN.pdf (accessed on 1 February 2022).

26. Erel, O. A novel automated direct measurement method for total antioxidant capacity using a new generation, more stable ABTS radical cation. Clin. Biochem. 2004, 37, 277-285. [CrossRef]

27. Wyatt, T.A.; Kharbanda, K.K.; Tuma, D.J.; Sisson, J.H. Malondialdehyde-acetaldehyde-adducted bovine serum albumin activates protein kinase $C$ and stimulates interleukin-8 release in bovine bronchial epithelial cells. Alcohol 2001, 25, 159-166. [CrossRef]

28. Armstrong, W.D.; Carr, C.W. Estimation of serum total protein. In Physiological Chemistry Laboratory Directions, 3rd ed.; Burges Publishing Co.: Minneapolis, MN, USA, 1964.

29. Doumas, B.T.; Peters, T. Serum and urine albumin: A progress report on their measurement and clinical significance. Clin. Chim. Acta 1997, 258, 3-20. [CrossRef]

30. Giangiacomo, J.; Cleary, T.G.; Cole, B.R.; Hoffsten, P.; Robson, A.M. Serum Immunoglobulins in the Nephrotic Syndrome. N. Engl. J. Med. 1975, 293, 8-12. [CrossRef]

31. Elias, M. Serum cortisol, testosterone, and testosterone-binding globulin responses to competitive fighting in human males. Aggress. Behav. 1981, 7, 215-224. [CrossRef]

32. Reitman, S.; Frankel, S. A Colorimetric Method for the Determination of Serum Glutamic Oxalacetic and Glutamic Pyruvic Transaminases. Am. J. Clin. Pathol. 1957, 28, 56-63. [CrossRef]

33. Kim, E.E.; Wyckoff, H.W. Reaction mechanism of alkaline phosphatase based on crystal structures: Two-metal ion catalysis. J. Mol. Biol. 1991, 218, 449-464. [CrossRef]

34. Eilers, R.J. Notification of Final Adoption of an International Method and Standard Solution for Hemoglobinometry Specifications for Preparation of Standard Solution. Am. J. Clin. Pathol. 1967, 47, 212-214. [CrossRef] [PubMed]

35. Jain, S.K. Membrane lipid peroxidation in erythrocytes of the newborn. Clin. Chim. Acta 1986, 161, 301-306. [CrossRef]

36. Kawahara, E.; Ueda, T.; Nomura, S. In Vitro Phagocytic Activity of White-Spotted Char Blood Cells after Injection with Aeromonas salmonicida Extracellular Products. Fish Pathol. 1991, 26, 213-214. [CrossRef]

37. OIE-World Organisation for Animal Health (OIE). Manual of Diagnostic Tests and Vaccines for Terrestrial Animals. 2021. Available online: https://www.oie.int/en/what-we-do/standards/codes-and-manuals/terrestrial-manual-online-access/ (accessed on 1 February 2022).

38. OIE-World Organisation for Animal Health (OIE). OIE Terrestrial Manual 2015; Chapter 2.3.4 Avian Influenza; OIE: Paris, France, 2015. 
39. Seal, B.S.; King, D.J.; Sellers, H.S. The avian response to Newcastle disease virus. Dev. Comp. Immunol. 2000, $24,257-268$. [CrossRef]

40. Cosgrove, A.S. An Apparently New Disease of Chickens: Avian Nephrosis. Avian Dis. 1962, 6, 385. [CrossRef]

41. Takatsy, G.; Hamar, M. The antibody content of immune sera absorbed with influenza virus, as determined by different serological tests. Acta Microbiol. Acad. Sci. Hung. 1956, 3, 299-306.

42. Balhaa, R.L.; Hinz, H.H.; Luders, H.; Siegmann, O. linical experiences with the drugs for lymphocyte transformation in chickens and turkey flocks. Tierarztl. Umsch. 1985, 43, 507-508.

43. Rainger, G.; Rowley, A. Antibacterial activity in the serum and mucus of rainbow trout, Oncorhynchus mykiss, following immunisation with Aeromonas salmonicida. Fish Shellfish. Immunol. 1993, 3, 475-482. [CrossRef]

44. Engstad, R.E.; Robertsen, B.; Frivold, E. Yeast glucan induces increase in lysozyme and complement-mediated haemolytic activity in Atlantic salmon blood. Fish Shellfish. Immunol. 1992, 2, 287-297. [CrossRef]

45. Allan, J.; Mitchell, T.; Harborne, N.; Bohm, L.; Crane-Robinson, C. Roles of H1 domains in determining higher order chromatin structure and H1 location. J. Mol. Biol. 1986, 187, 591-601. [CrossRef]

46. Biswas, H.R.; Haoque, M.M.; Oxley, M.; Prodhan, M.A.M. A comparative study on the protection of indigenous chickens against Newcastle disease induced by Australian NDV4HR and locally produced conventional vaccines in Bangladesh. Prev. Vet. Med. 1996, 26, 157-164. [CrossRef]

47. Culling, C.F. Handbook of Histopathological and Histochemical Staining Techniques, 3rd ed.; Butterworth: London, UK, 1983.

48. SAS Intstitute Inc. SAS/STAT Software, Version 9; SAS Institute, Inc.: Cary, NC, USA, 2002.

49. Hewson, K.; Liu, S.; Morgan, N.; Selle, P.; Wu, S.; Townsend, G. Agrifutures Australia Chicken Meat-Nutrition Program of Research. In Proceedings of the 31st Annual Australian Poultry Science Symposium, Sydney, Australia, 16-19 February 2020; p. 58

50. Al-Khalaifah, H.; Al-Nasser, A.; Abdulmalek, N.; Al-Mansour, H.; Ahmed, A.; Ragheb, G. Impact of SARS-Con-V2 on the poultry industry in Kuwait: A case study. Front. Vet. Sci. 2002, 7, 656. [CrossRef]

51. Onifade, A.A. Growth performance, carcass characteristics, organs measurement and haematology of broiler chickens fed a high fibre diet supplemented with antibiotics or dried yeast. Food/Nahrung 1997, 41, 370-374. [CrossRef]

52. Attia, Y.; Hassan, R.A.; El-Din, A.E.T.; Abou-Shehema, B.M. Effect of ascorbic acid or increasing metabolizable energy level with or without supplementation of some essential amino acids on productive and physiological traits of slow-growing chicks exposed to chronic heat stress. J. Anim. Physiol. Anim. Nutr. 2010, 95, 744-755. [CrossRef] [PubMed]

53. Abudabos, A.M. Effect of Enzyme Supplementation to Normal and Low Density Broiler Diets Based on Corn-soybean Meal. Asian J. Anim. Veter. Adv. 2012, 7, 139-148. [CrossRef]

54. Attia, Y.A. Value of rice bran, its maximal utilization, and upgrading by phytase and other enzymes and diet-formulation based on available amino acid for broiler chicks. Arch. Geflugelkd. 2003, 67, 157-166.

55. Steenfeldt, S. The dietary effect of different wheat cultivars for broiler chickens. Br. Poult. Sci. 2001, 42, 595-609. [CrossRef]

56. Yamauchi, K.-E. Review on Chicken Intestinal Villus Histological Alterations Related with Intestinal Function. J. Poult. Sci. 2002, 39, 229-242. [CrossRef]

57. Iji, P. The impact of cereal non-starch polysaccharides on intestinal development and function in broiler chickens. World's Poult. Sci. J. 1999, 55, 375-387. [CrossRef]

58. Thomas, D.V.; Ravindran, V. Effect of Cereal Type on the Performance, Gastrointestinal Tract Development and Intestinal Morphology of the Newly Hatched Broiler Chick. J. Poult. Sci. 2008, 45, 46-50. [CrossRef]

59. Akhtar, M.; Tariq, A.F.; Awais, M.M.; Iqbal, Z.; Muhammad, F.; Shahid, M.; Hiszczynska-Sawicka, E. Studies on wheat bran Arabinoxylan for its immunostimulatory and protective effects against avian coccidiosis. Carbohydr. Polym. 2012, 90, 333-339. [CrossRef] [PubMed]

60. Lin, W.C.; Lee, M.T.; Lo, C.T.; Chang, S.C.; Lee, T.-T. Effects of dietary supplementation of Trichoderma pseudokoningii fermented enzyme powder on growth performance, intestinal morphology, microflora and serum antioxidantive status in broiler chickens Ital. J. Anim. Sci. 2018, 17, 153-164. [CrossRef]

61. Li, B.; Leblois, J.; Taminiau, B.; Schroyen, M.; Beckers, Y.; Bindelle, J.; Everaert, N. The effect of inulin and wheat bran on intestinal health and microbiota in the early life of broiler chickens. Poult. Sci. 2018, 97, 3156-3165. [CrossRef]

62. Al-Khalaifa, H.; Al-Nasser, A.; Al-Surayee, T.; Al-Kandari, S.; Al-Enzi, N.; Al-Sharrah, T.; Ragheb, G.; Al-Qalaf, S.; Mohammed, A. Effect of dietary probiotics and prebiotics on the performance of broiler chickens. Poult. Sci. 2019, 98, 4465-4479. [CrossRef] [PubMed]

63. Cao, Y.; Xu, Y.; Auchoybur, M.L.; Chen, W.; He, S.; Qin, W.; Su, C.; Huang, F.; Qiu, Z.; Li, L.; et al. Regulatory role of IKK $\alpha$ in myocardial ischemia/reperfusion injury by the determination of M1 versus M2 polarization of macrophages. J. Mol. Cell. Cardiol. 2018, 123, 1-12. [CrossRef]

64. Korte, J.; Fröhlich, T.; Kohn, M.; Kaspers, B.; Arnold, G.J.; Härtle, S. 2D DIGE analysis of the bursa of Fabricius reveals characteristic proteome profiles for different stages of chicken B-cell development. Proteomics 2013, 13, 119-133. [CrossRef]

65. Nilson, A.P.; Miazzo, R.D. Use of Brewers Yeast S. cerevisiae to Replace Part of the Vitamin Mineral Premix in Finisher Broiler Diets; XXII Worlds Poult. Cong.: Istanbul, Turkey, 2004.

66. Shareef, A.M.; Al-Dabbagh, A.S.A. Effect of probiotic (Saccharomyces cerevisiae) on performance of broiler chicks. Iraqi J. Vet. Sci. 2009, 23 (Suppl. S1), 23-29. 
67. Koc, F.; Samli, H.; Okur, A.; Ozduven, M.; Akyurek, H.; Senkoylu, N. Effects of Saccharomyces cerevisiae and/or mannanoligosaccharide on performance, blood parameters and intestinal microbiota of broiler chicks. Bulg. J. Agric. Sci. 2010, 16, 643-650.

68. Abou El-Naga, M.K. Effect of dietary yeast supplementation on broiler performance. Egypt. Poult. Sci. J. 2012, 32, 95-106.

69. Valdivie, M. Saccharomyces yeast as a by-product from alcohol production on final molasses in diets for broilers. Cuban J. Agric. Sci. 1975, 9, 327-331.

70. Zhang, A.; Lee, B.; Lee, S.; Lee, K.; An, G.; Song, K.; Lee, C. Effects of yeast (Saccharomyces cerevisiae) cell components on growth performance, meat quality, and ileal mucosa development of broiler chicks. Poult. Sci. 2005, 84, 1015-1021. [CrossRef] [PubMed]

71. Paryad, A.; Mahmoudi, M. Effect of different levels of supplemental yeast Saccharomyces cerevisiae on performance, blood constituents and carcass characteristics of broiler chicks. Afr. J. Agric. Res. 2008, 3, 835-842.

72. Hosseini, S. The Effect of Saccharomysec cerevisiae on blood parameters of broiler chicken's. Glob. Vet. 2011, 74, 411-414.

73. Newman, K. Mannan-oligosaccharides: Natural polymers with significant impact on the gastrointestinal microflora and the immune system. Biotechnol. Feed. Ind. 1994, 10, 167-180.

74. Spring, P.; Wenk, C.; Dawson, K.A.; Newman, K.E. The effects of dietary mannanoligosaccharides on cecal parameters and the concentrations of enteric bacteria in the ceca of salmonella-challenged broiler chicks. Poult. Sci. 2000, 79, 205-211. [CrossRef] [PubMed]

75. Saleh El-Din, S.H.S.; Abd El-Hamid, A.E. Effect of different levels of vitamin E and mannanoligosaccharide on productive performance and immune response of broiler chicks. Poult. Sci. 2012, 91, 30.

76. Attia, Y.; Al-Khalaifah, H.; El-Hamid, H.A.; Al-Harthi, M.; El-Shafey, A. Growth performance, digestibility, intestinal morphology, Carcass traits and meat quality of broilers fed marginal nutrients deficiency-diet supplemented with different levels of active Yeast. Livest. Sci. 2020, 233, 103945. [CrossRef]

77. Bilal, R.M.; Hassan, F.U.; Saeed, M.; Rafeeq, M.; Zahra, N.; Fraz, A.; Saeed, S.; Khan, M.A.; Mahgoub, H.A.M.; Farag, M.R.; et al. Role of Yeast and Yeast-Derived Products as Feed Additives in Broiler Nutrition. Anim. Biotechnol. 2021, 1-10. [CrossRef]

78. He, T.; Mahfuz, S.; Piao, X.; Wu, D.; Wang, W.; Yan, H.; Ouyang, T.; Liu, Y. Effects of live yeast (Saccharomyces cerevisiae) as a substitute to antibiotic on growth performance, immune function, serum biochemical parameters and intestinal morphology of broilers. J. Appl. Anim. Res. 2021, 49, 15-22. [CrossRef] 